\title{
PHOTOMETRIC AND STRUCTURAL PROPERTIES OF NGC 6544: A COMBINED VVV-HUBBLE SPACE TELESCOPE STUDY
}

\author{
Roger E. Cohen ${ }^{1}$, Francesco Mauro ${ }^{1}$, Doug Geisler ${ }^{1}$, Christian Moni Bidin ${ }^{2}$, \\ Aaron DotTer $^{3}$, And Charles Bonatto ${ }^{4}$ \\ ${ }^{1}$ Departamento de Astronomía, Universidad de Concepción, Casilla 160-C, Concepción, Chile \\ 2 Instituto de Astronomía, Universidad Católica del Norte, Av. Angamos 0610, Antofagasta, Chile \\ ${ }^{3}$ Research School of Astronomy and Astrophysics, The Australian National University, Canberra ACT 2611, Australia \\ ${ }^{4}$ Departamento de Astronomia, Universidade Federal de Rio Grande do Sul, Av. Bento Gonçalves 9500, Porto Alegre 91501-970, RS, Brazil \\ Received 2013 November 20; accepted 2014 March 7; published 2014 June 10
}

\begin{abstract}
We combine archival Hubble Space Telescope imaging with wide-field near-infrared photometry to study the neglected metal-poor Galactic globular cluster NGC 6544. A high spatial resolution map of differential reddening over the inner portion of the cluster is constructed, revealing variations of up to half of the total reddening, and the resulting corrected color-magnitude diagrams reveal a sparse blue horizontal branch and centrally concentrated blue straggler population, verified via relative proper motions. Using the corrected photometry to investigate the cluster distance, reddening, and age via direct comparison to well-calibrated photometry of clusters with similar metallicities, we estimate $(m-M)_{0}=11.96, E(B-V)=0.79$, and an age coeval with M13 to within the relevant uncertainties. Although our data are insufficient to place tight constraints on the reddening law toward NGC 6544, we find no strong evidence that it is non-standard at optical or near-infrared wavelengths. We also provide near-infrared fiducial sequences extending nearly 2 mag below the cluster main sequence turnoff, generated from a statistically decontaminated sample of cluster stars. Lastly, we redetermine the cluster center and construct a radial number density profile which is well fit by an atypically flat power law with a slope of about 1.7. We discuss this result, together with a flattened main sequence luminosity function and inverted mass function, in the context of mass segregation and tidal stripping via interactions with Milky Way potential.
\end{abstract}

Key words: Galaxy: evolution - globular clusters: general - globular clusters: individual (NGC 6544)

Online-only material: color figures, supplemental data

\section{INTRODUCTION}

Clues to the formation and evolution of the Milky Way are imprinted in the stellar populations of Galactic globular clusters (GGCs). The GGCs located in the direction of the Milky Way bulge have largely escaped the level of scrutiny directed toward the GGC population at large since the advent of recent spacebased imaging surveys (e.g., Piotto et al. 2002; Sarajedini et al. 2007) due at least in part to strong and differential extinction. This is true not only of clusters physically located in the inner bulge, but also of those in the bulge direction, where the combination of field star contamination and ubiquitous reddening variations can render photometric analyses daunting. NGC 6544 is perhaps the best example of such a circumstance, since although it lies at a distance of $R_{\odot} \lesssim 3 \mathrm{kpc}$, it has managed to largely evade detailed study.

The first photometric study of NGC 6544, by Alcaino (1983), obtained $U B V$ photoelectric photometry down to the horizontal branch (HB) and estimated $E(B-V)=0.70$, a galactocentric distance of $R_{\mathrm{GC}}=6.1 \mathrm{kpc}$, and a heliocentric distance of $R_{\odot}=$ $2.9 \mathrm{kpc}$. Webbink (1985) lists $E(B-V)=0.73, R_{\mathrm{GC}}=6.2 \mathrm{kpc}$, and $R_{\odot}=2.6 \mathrm{kpc}$, as well as approximate core and tidal radii of 0.47 and 11.75 , which we will show are likely much closer to the true value than a more recent estimate. Hazen (1993) conducted a variability study using 29 photographic plates, identifying a candidate fundamental mode RR Lyrae variable and a candidate eclipsing binary within the cluster. NGC 6544 was also included in the GGC surveys of Rosenberg et al. (2000) and Piotto et al. (2002), although their results come with the caveat that differential reddening could cause significant uncertainties in derived parameters.
Fortunately, $[\mathrm{Fe} / \mathrm{H}]$ seems to be fairly well determined for NGC 6544. Carretta et al. (2009) list $[\mathrm{Fe} / \mathrm{H}]=-1.47 \pm 0.07$, in excellent agreement with the value of $[\mathrm{Fe} / \mathrm{H}]=-1.43 \pm 0.14$ obtained on the same scale by Saviane et al. (2012) using Ca II triplet spectroscopy. Valenti et al. (2010) list a slightly higher value of $[\mathrm{Fe} / \mathrm{H}]=-1.36$ (after transforming to the Carretta et al. 2009 scale) from near-infrared photometric calibrations using the technique described in Ferraro et al. (2006), although this difference is well within their estimated systematic uncertainty of 0.2 dex. In terms of the global metallicity defined by Salaris et al. $(1993)$ as $[\mathrm{M} / \mathrm{H}]=[\mathrm{Fe} / \mathrm{H}]+\log \left(0.638 f_{\alpha}+0.362\right)$, where $f_{\alpha}=10^{[\alpha / \mathrm{Fe}]}$, the Carretta et al. (2009) value of $[\mathrm{Fe} / \mathrm{H}]$ and the linear $[\alpha / \mathrm{Fe}]$ versus $[\mathrm{Fe} / \mathrm{H}]$ relation of Nataf et al. (2013b) give $[\alpha / \mathrm{Fe}]=0.36 \pm 0.06$ and $[\mathrm{M} / \mathrm{H}]=-1.21 \pm 0.08$.

Our motivation for better characterizing NGC 6544 is not solely due to the lack of recent photometric studies. Additionally, it was apparent from wide-field near-infrared imaging (described in the next section) that this cluster may be significantly more spatially extended than literature values imply. Furthermore, its proximity presents the advantage that the difference in line of sight reddening with respect to the Galactic bulge allows the cluster population to be isolated in color-magnitude diagrams (CMDs) alone. In light of the ensemble of ground- and space-based photometry now available for NGC 6544, we decided to undertake a more detailed study of both its photometric and structural properties.

This paper is organized as follows: in the next section, we describe our photometric data sets and their respective reduction processes. In Section 3, we generate and apply a differential reddening map before discussing the various corrected CMDs, including different subsamples selected via relative proper 
Table 1

Archival HST Photometry

\begin{tabular}{llccccc}
\hline \hline Target & Instrument & Exposures & Filters & Program & PI \\
\hline NGC 6544 & WFPC2 & $40 \mathrm{~s}+2 \times 100 \mathrm{~s}, 3 \mathrm{~s}+2 \times 30 \mathrm{~s}$ & $F 439 W, F 555 W$ & 8118 & Piotto \\
NGC 6544 & ACS WFC & $120 \mathrm{~s}+680 \mathrm{~s}, 20 \mathrm{~s}$ & $F 435 W, F 625 W$ & 10349 & Lewin \\
NGC 6981 & WFPC2 & $40 \mathrm{~s}+2 \times 160 \mathrm{~s}, 10 \mathrm{~s}+40 \mathrm{~s}$ & $F 439 W, F 555 W$ & 7470 & King \\
NGC 5904 & ACS WFC & $70 \mathrm{~s}+680 \mathrm{~s}, 300 \mathrm{~s}$ & $F 435 W, F 625 W$ & 10120 & Anderson \\
NGC 6205 & ACS WFC & $120 \mathrm{~s}+680 \mathrm{~s}, 20 \mathrm{~s}+360 \mathrm{~s}$ & $F 435 W, F 625 W$ & 10349 & Lewin \\
\hline
\end{tabular}

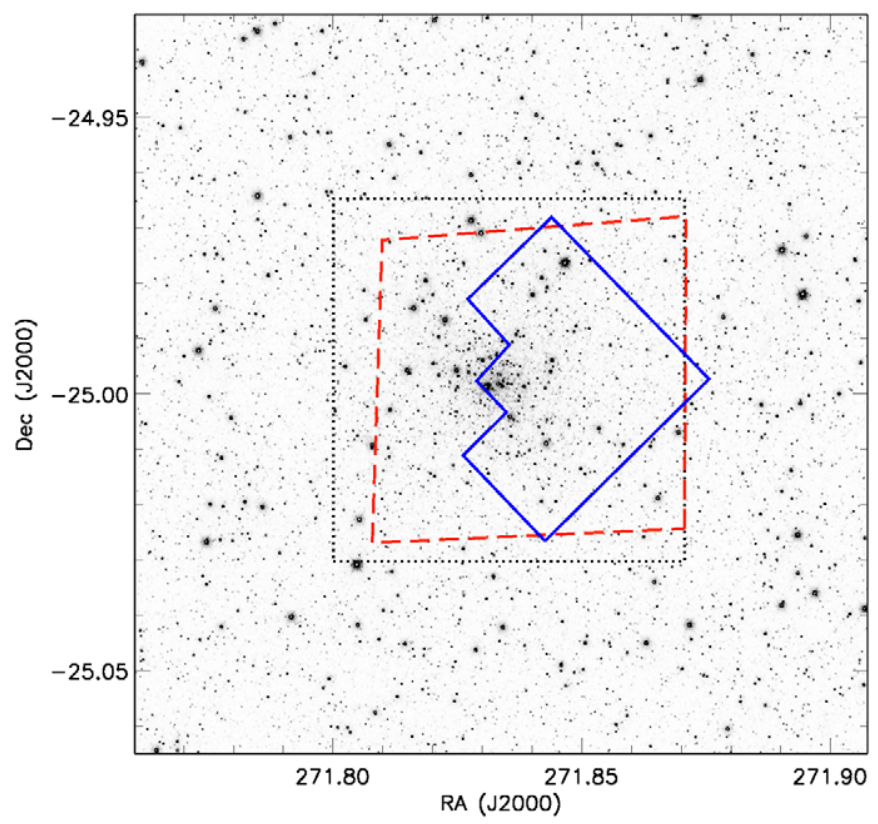

Figure 1. An $8^{\prime} \times 8^{\prime}$ portion of a VVV $K_{S}$ image centered on NGC 6544 , showing the ACS, Rosenberg et al., and WFPC2 fields of view as dashed, dotted, and solid lines, respectively. North is up and east is to the right.

(A color version of this figure is available in the online journal.)

motions as well as a statistical field. In Section 4, we discuss the age of NGC 6544, and in Section 5 we estimate the cluster distance modulus and reddening, both via empirical comparison to other GGCs. In Section 6, we discuss the reddening law toward NGC 6544 in light of our various sources of optical and infrared photometry, and in Section 7 we present cluster luminosity and mass functions (MFs). In Section 8, we analyze of the structural properties of the cluster, redetermining its center and constructing a number density profile, and in the final section we summarize our results and suggest directions for further investigation.

\section{OBSERVATIONS AND DATA REDUCTION}

The observations of NGC 6544 that we employ come from several sources. The first is a wide-field ground-based nearinfrared survey, described in Section 2.1. We supplement these observations with ground-based $V, I$ photometry from Rosenberg et al. (2000), and also present photometry carried out using archival Hubble Space Telescope (HST) images obtained with the Advanced Camera for Surveys (ACS) WideField Camera (WFC) using the $F 435 W$ and $F 625 W$ filters (GO-10439, PI: Lewin) and WFPC2 in the $F 439 W$ and $F 555 W$ filters (SNAP-8118, PI: Piotto). We summarize all of the archival HST observations employed here, including those of other clusters which we use for empirical comparisons in Section 5.1, in Table 1. The fields of view of all of our ground- and space-based NGC 6544 observations are shown in Figure 1.

\subsection{VVV Photometry}

The Vista Variables in the Via Lactea (VVV; Minniti et al. 2010; Catelan et al. 2011), an ESO public survey, is obtaining $Y Z J H K_{S}$ imaging over a $562 \mathrm{deg}^{2}$ field including the Galactic bulge and a portion of the disk. Observations are carried out at the $4 \mathrm{~m}$ Visible and Infrared Survey Telescope for Astronomy (VISTA), equipped with the VIRCAM imager (Emerson et al. 2006). This detector is comprised of a $4 \times 4$ array of $162048 \times$ 2048 chips with a pixel size of 0.34 , and the details of the telescope, instrument, and survey strategy can be found in Saito et al. (2012, and references therein). For the present study, 130 individual chips from 30 images were employed. Pre-processing of VVV images is accomplished at the Cambridge Astronomical Survey Unit (CASU), and we have carried out point-spread function (PSF) photometry on CASU-preprocessed images using the VVV-SkZ pipeline (Mauro et al. 2013). This pipeline is designed to automate the processing of CASU images to obtain instrumental PSF photometry in crowded fields via the iterative usage of the DAOPHOT/ALLFRAME suite (Stetson 1987, 1994). We have chosen to perform photometric and astrometric calibration to the Two Micron All Sky Survey (2MASS; Skrutskie et al. 2006), also employing the VVV-SkZ pipeline. Each of the $J H K_{S}$ magnitudes are transformed to the 2MASS system using the classical linear transformation equations of the form $m_{2 \mathrm{MASS}}-m_{\text {inst }}=a+b\left(J-K_{S}\right)_{2 \mathrm{MASS}}$, where $a$ is a zero point offset and $b$ is a linear color term. The coefficients $a$ and $b$ are obtained using least-squares fitting, but in lieu of a sigma clip, a weighting scheme is used that downweights the most discrepant data points. ${ }^{5}$ Only standards within a pre-defined magnitude range are used as local calibrators, since they must lie faintward of the VVV saturation limit while also avoiding the faint limit imposed by crowding in 2MASS. Therefore we have used only stars with $10.6 \leqslant J \leqslant$ $12.8,10.0 \leqslant H \leqslant 12.0,9.5 \leqslant K_{S} \leqslant 12.0$ to calibrate our VISTA photometry to the 2MASS system. Furthermore, stars with nearby companions which contribute non-negligibly to their measured magnitudes are rejected from the calibration process (see Mauro et al. 2013 for details), and the resultant photometric zero points have $1 \sigma$ uncertainties of less than $0.02 \mathrm{mag}$. Astrometric calibration has been performed to the coordinates given in the $2 \mathrm{MASS}$ point source catalog using the World Coordinate System (WCS) given by CASU in the VVV image headers as an initial guess. In accord with the astrometric precision of 2MASS, the resulting astrometric transformation has an rms positional residual of $\sim 0^{\prime} .2$ with respect to $2 \mathrm{MASS}$. A more extensive discussion regarding the calibration of VVV to 2MASS photometry can be found in Chené et al. (2012), and further details and examples can be found in Moni Bidin et al. (2011). Since our VVV photometry becomes unusable below

\footnotetext{
5 The algorithm is based on a series of lectures presented at "V Escola Avancada de Astrofisica" by P. B. Stetson, see

http://ned.ipac.caltech.edu/level5/Stetson/Stetson_contents.html and http://www.cadc.hia.nrc.gc.ca/community/STETSON/homogeneous/.
} 

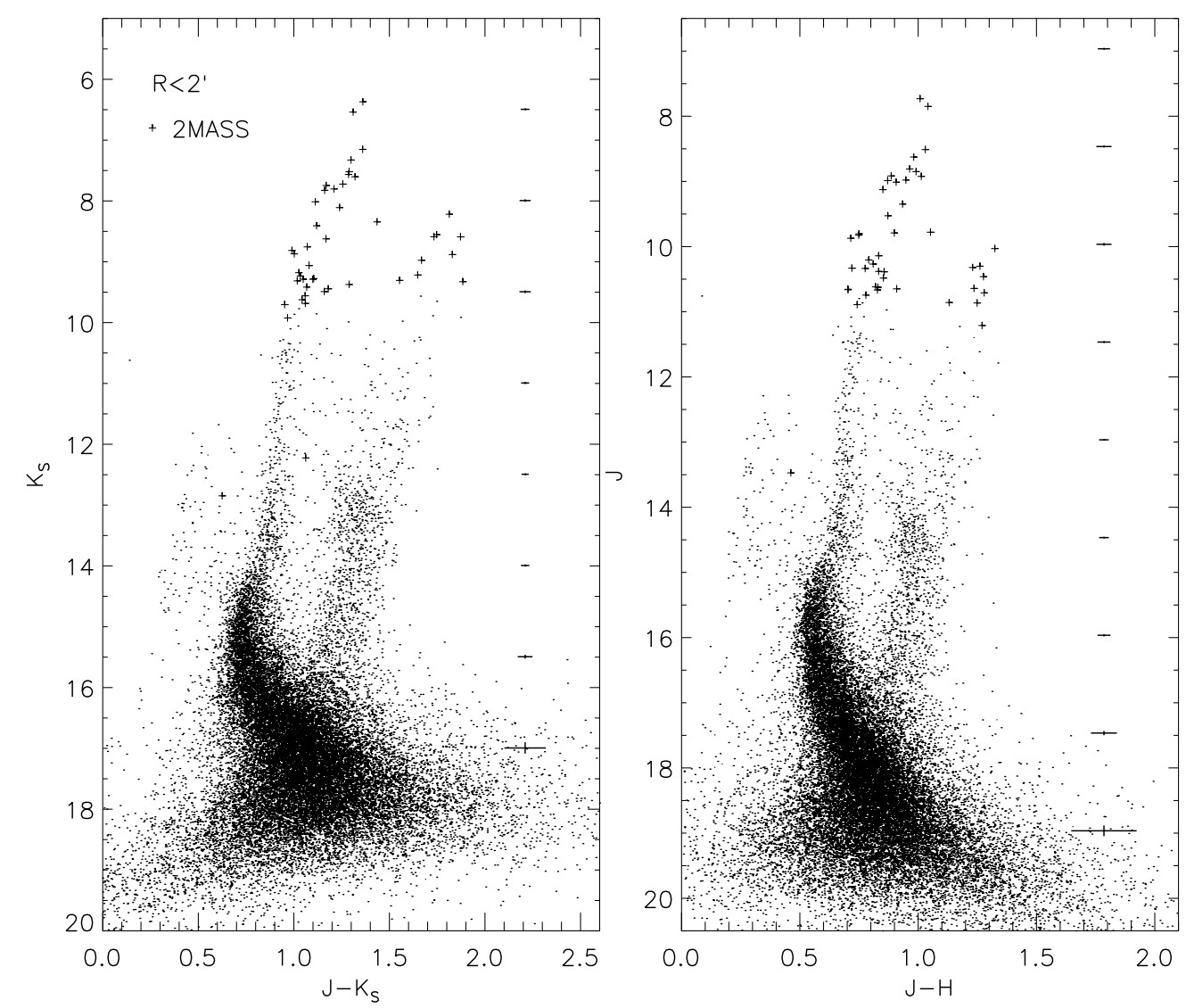

Figure 2. Color-magnitude diagrams of the VVV photometry in the $\left(K_{S}, J-K_{S}\right)$ (left) and $(J, J-H)$ (right) planes, showing stars within $2^{\prime}$ of the cluster center. Stars with photometry taken directly from 2MASS are shown as plus symbols, and median photometric errors in magnitude bins are shown along the right-hand side of each plot.

the tip of the red giant branch (RGB) due to saturation, we supplement these data with $J H K_{S}$ photometry from 2MASS for the brightest stars, indicated in the CMDs in Figure 2.

\subsection{HST Photometry}

We obtained PSF photometry from the ACS WFC images using the latest version of DOLPHOT $2.0^{6}$ (Dolphin 2000) largely according to the author's prescriptions. In processing individual images, we set ncombine $=2$ where appropriate in order to obtain correct signal to noise values, and found that setting Force1 $=0$ resulted in photometry more easily cleaned of spurious detections than setting Force $1=1$, which forces all objects to be categorized as stellar when performing PSF photometry. Next, we cut our catalog to only well measured stars by retaining from the DOLPHOT output only those sources with object type $=0,-0.5<$ sharpness $<0.5$, roundness $<1$, a value of zero for the photometric quality flags in both filters, and a crowding parameter $<0.2$. We found that the crowding cut was the most reliable way to remove spurious detections caused by the many oversaturated stars and their diffraction spikes in the ACS field of view, and the small fraction of real stars that are also lost can be well quantified by artificial star tests.

Artificial star tests were performed to quantify photometric incompleteness as a function of both magnitude and radial distance from the cluster center using the fakestars module of DOLPHOT. In order to obtain adequate Poissonian statistics for the artificial stars, we performed 10 fakestars runs, each

\footnotetext{
6 http://americano.dolphinsim.com/dolphot
}

with 30,000 input stars. To generate the input positions and magnitudes for the artificial stars, the fakelist task was used, employing the -usexy option with the coordinates of the real stars as input so that the artificial stars are similarly concentrated toward the cluster center, and the -usecmd option was used with the cluster fiducial sequence (evenly spaced in magnitude) as input (see Section 3.1) so that the artificial stars occupy approximately the same CMD region as real cluster stars (albeit with some scatter, as the fakestars task uses a fixed CMD box size). Each input star was considered recovered if it passed all of the selection cuts applied to real stars described above. The resulting CMD of the 32,851 stars passing our selection cuts, as well as a map of photometric completeness in the magnitude-radius plane, is shown in Figure 3.

We verify the astrometric calibration of the ACS photometry (obtained from the WCS information in the.drz image header), which is typically accurate to a few tenths of an arcsecond (Gonzaga et al. 2011), via comparison to 2MASS and VVV. Using 2MASS alone, we were able to match only 118 stars since there is a narrow luminosity range between the bright saturation limit of the ACS photometry and the faint limit of 2MASS, finding offsets of $(\Delta$ R.A. $\cos \delta, \Delta$ decl. $)=(-0$ ' 17 , $0.41) \pm\left(0^{\prime \prime} 33,0^{\prime \prime} 37\right)$ in the sense (2MASS-ACS). However, matching the ACS photometry to our VVV photometry, which is astrometrically calibrated to 2MASS, we were able to match $92.4 \%$ of the VVV sources in the ACS field of view, finding offsets of $(\Delta$ R.A. $\cos \delta, \Delta$ decl. $)=\left(-0^{\prime \prime} 23,-0.56\right)$ with an astrometric rms of $0^{\prime \prime} 23$. These offsets are well within the uncertainties from the direct comparison to 2MASS, and similar 

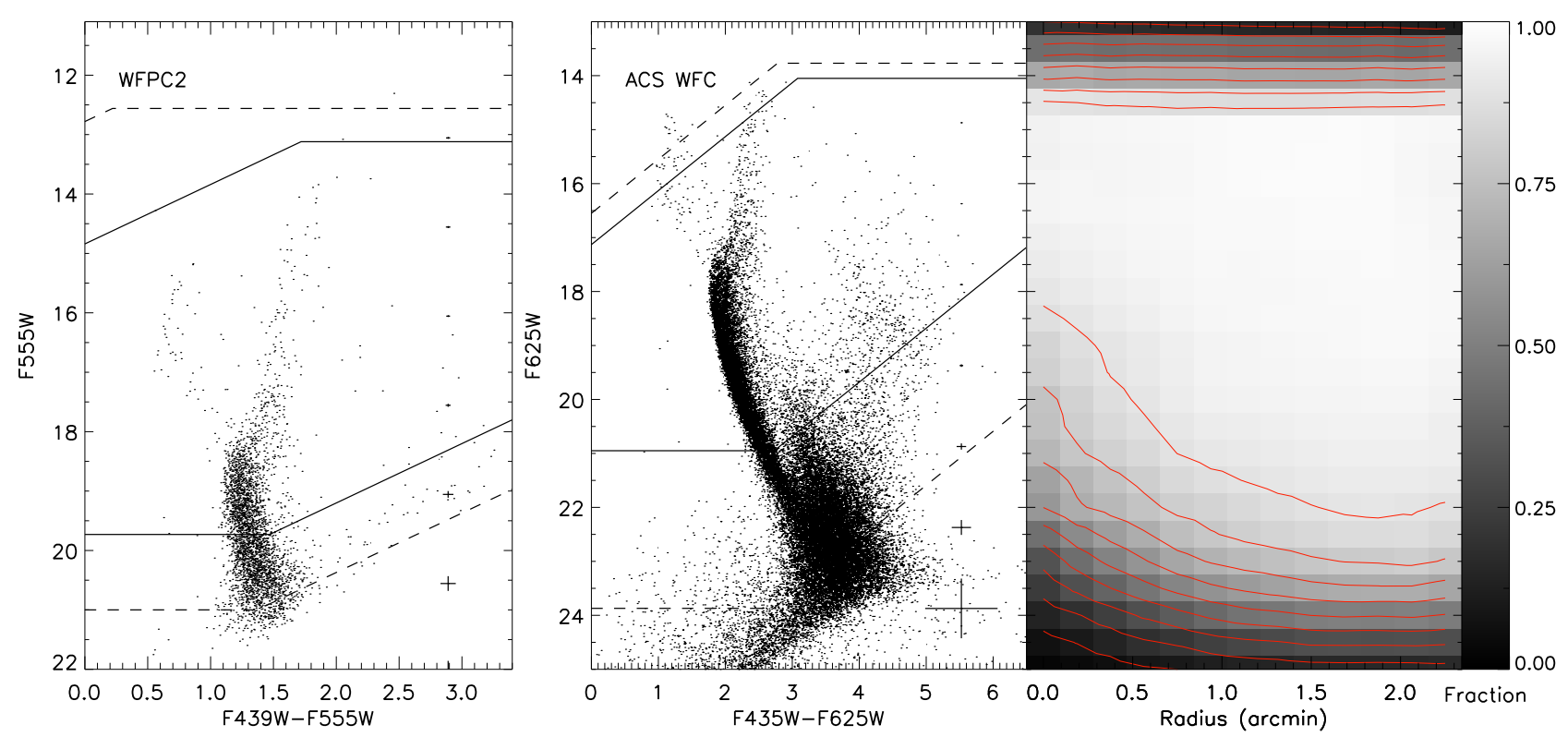

Figure 3. Left: color-magnitude diagram showing all stars which passed our quality cuts for the WFPC 2 photometry. Median color and magnitude errors in magnitude bins resulting from artificial star tests are shown along the right-hand side, and 50\% and 90\% completeness limits are indicated by dashed and solid lines respectively. Center: same, but for the ACS WFC photometry. Right: a map of the ACS photometric completeness fraction in the $F 625 W$-clustercentric radius plane. Contours in intervals of 0.1 in completeness fraction are overplotted.

(A color version of this figure is available in the online journal.)

(and larger) offsets between ACS header astrometry and 2MASS have been found before (Anderson et al. 2008).

The WFPC2 images were processed in a similar manner as the ACS images using the most recent version of HSTPHOT, ${ }^{7}$ which is optimized to perform PSF photometry on WFPC2 images. Similar quality cuts were made in the final catalog as for the ACS photometry, except that on the advice of the DOLPHOT manual, we required $\chi<1.5$ and no crowding cut, as we found spurious detections to be less problematic. Astrometric calibration was performed using the WCS in the image headers and applying the geometric distortion solution of Anderson \& King (2003), and the astrometric solution is compared to that from the ACS photometry in order to measure relative proper motions in Section 3.3. However, since only the planetary camera (PC) chip was useful for this purpose, we also compared the coordinates from the wide-field (WF) chips to the ACS photometry as a further check of the astrometric precision of WFPC2, finding an astrometric rms of $\leqslant 0$ '. 16 with respect to the ACS astrometry for all WFPC 2 chips. The CMD of 7698 stars passing our cuts and the completeness limits resulting from artificial star tests using HSTPHOT are shown in Figure 3.

\section{COLOR-MAGNITUDE DIAGRAMS}

\subsection{Reddening Map}

It is clear from the CMDs of Figures 2 and 3 that differential reddening is significant on small spatial scales, as has been found for other GGCs, particularly in the direction of the Galactic bulge (e.g., Alonso Garcia et al. 2012; Massari et al. 2012). For this reason, we use the ACS photometry to construct a reddening map in the inner region of the cluster before further discussing the morphology of the NGC 6544 CMDs which we obtain from the various sources of imaging described in the previous section.

The differential reddening corrections and uncertainties are measured using a method originally presented by von Braun \&

\footnotetext{
7 http://purcell.as.arizona.edu/hstphot
}

Mateo (2001) in which the reddening correction at the physical position of a particular star is quantified by the shift in the CMD parallel to the direction of the reddening vector required to place the star on a fiducial cluster reference sequence. In practice, we obtain this measurement by rotating the CMD to be perpendicular to the reddening vector (e.g., Milone et al. 2012).

We construct the final reddening map using an iterative technique, and to generate the reddening map for the first iteration, we use a set of reference stars with $17.5 \leqslant F 625 W \leqslant 20.5$ and $1.5 \leqslant(F 435 W-F 625 W) \leqslant 2.8$. The color limit mitigates the influence of field stars, especially those fainter than $F 625 W \sim 20$ with $3<(F 435 W-F 625 W)<4$, and the bright magnitude limit guards against the influence of cluster HB stars, blue stragglers, and field stars that lie brightward of the main sequence turnoff. Meanwhile, the faint magnitude limit, in addition to effectively serving as a signal-to-noise cut, avoids the faintest part of the observed cluster sequence, which becomes nearly parallel to the reddening vector. The first reddening map iteration employs 6432 reference stars in the aforementioned magnitude range, and after the first iteration, stars are used as reference stars only if they fall inside the aforementioned magnitude limits and lie within $5 \sigma$ of the fiducial sequence (generated from the most recent iteration as described below) in color. By using the cluster main sequence to construct a reddening map, we can exploit the most well populated region of the cluster CMD to maximize spatial resolution while having the cluster giant branch and HB available as gauges of the effectiveness of the dereddening procedure. The reddening map is constructed and improved iteratively, using the following steps.

1. First, a reference fiducial sequence is constructed. This itself is done iteratively, wherein the CMD is divided into magnitude bins of width 0.2 mag and the median color in each bin is obtained, rejecting stars with colors more than $2 \sigma$ from the median in each bin. An initial rough fiducial sequence is constructed by applying a low order polynomial smoothing to the resulting median bin colors as a function of their magnitude. 
Next, this procedure is repeated, but obtaining the median color in $0.1 \mathrm{mag}$ intervals and rotating the CMD of each bin so that it is parallel to the fiducial sequence from the previous iteration (e.g., Marin-Franch et al. 2009; Milone et al. 2012). The median color is then derotated back to the original frame and a new fiducial sequence is constructed, fitting points along the RGB with a third-order polynomial since it is relatively sparsely populated. In this way, the fiducial sequence is recalculated until convergence is indicated by the total change in the median bin colors remaining constant to within 0.01 since the previous iteration. The use of a fiducial sequence that is empirically determined after each dereddening iteration has the advantage that it does not require an assumption a priori of any particular set of evolutionary models or cluster parameters.

2. The CMD is rotated to be perpendicular to the reddening vector to measure the distance from each reference star to the fiducial sequence along the reddening vector.

3. The value of the reddening correction (and uncertainty) that is assigned to the spatial location of each reference star is the sigma-clipped weighted mean (and standard deviation) of the individual reddening corrections, taken among the 20 nearest reference stars. The use of a fixed number of neighbors rather than a fixed spatial box size has the advantage that it gives a characteristic spatial resolution for each reddening value (which we refer to as bandwidth) and allows a higher spatial resolution in more crowded regions (e.g., Alonso Garcia et al. 2012; Milone et al. 2012). The uncertainty on the reddening value for each reference star is calculated from the first and last contact points where the photometric error ellipse of the star contacts the fiducial sequence while being slid along the reddening vector in the CMD. Rather than assuming the error in color as the sole source of photometric uncertainty when calculating the uncertainty of the reddening correction, we note that color and magnitude errors may be correlated and conservatively (over)estimate the photometric uncertainty along the horizontal CMD axis for each star as $\sigma_{c^{\prime}}=\sqrt{\sigma_{c}^{2}+\sigma_{m}^{2}}$, where $\sigma_{c}$ and $\sigma_{m}$ are the photometric uncertainty in color and magnitude, respectively. The uncertainty of the reddening correction in the $(F 625 W, F 435 W-F 625 W)$ plane is then $\sigma_{A}=\left(\sigma_{c^{\prime}} /\left|\cos \left(\theta_{A}\right)\right|\right)$, where $\theta_{A}$ is the angle between the reddening vector and the horizontal axis of the CMD. The uncertainty of the reddening correction in terms of $E(B-V)$ is then $\sigma_{E(B-V)}=\sin \theta_{A}\left(A_{F 625 W} / E(B-V)\right)^{-1}$, where we have assumed $R_{V}=3.1$ and $\left(A_{F 435 W} / A_{V}, A_{F 625 W} / A_{V}\right)=$ $(1.337,0.850)$ (see Section 6.2). The weighting of the individual reddening corrections using their uncertainties, together with the use of a sigma clip, mitigates the influence of outliers and field stars.

4. The resulting reddening corrections and their uncertainties as a function of spatial location, determined from the reference stars, are applied to all stars by interpolating two dimensionally in $(\Delta$ R.A. $\cos \delta, \Delta$ decl.) space among the reference star reddening values determined in the previous steps.

5. Steps 1-4 are repeated until the median ratio between the residual reddening correction since the last iteration and the uncertainty in the reddening correction taken across all stars decreases below one, typically in 2-4 iterations. This iterative process is crucial to maximizing the precision and spatial resolution of the final map, as the corrections from each iteration are applied to all stars within the spatial region encompassed by the reddening map (although since our map is generated using the ACS photometry, it is necessarily spatially restricted to the ACS field of view) before regenerating an improved empirical fiducial sequence. The final reddening correction for each star is the sum over all iterations of the corrections determined in step 3 (if the star is a reference star) or step 4 (if the star is not a reference star).

As an additional test of the dereddening procedure, we performed the entire dereddening process described above in the $(F 435 W, F 435 W-F 625 W)$ plane as well. The difference in the resulting reddening corrections $\Delta E(B-V)$ between the two maps, taken across all stars, has a mean of -0.009 and a standard deviation of 0.011 , so to obtain our final map, shown in Figure 4, the two were averaged.

We also tested the sensitivity of the dereddening procedure to our assumption of the total to selective extinction $R_{V}$ by generating a reddening map assuming extinction coefficients appropriate for $R_{V}=2.8$ (see Section 6.2). The corrections seem to be fairly insensitive to this choice: the difference in the corrected $F 625 \mathrm{~W}$ magnitude has a mean and standard deviation of 0.008 and 0.027 , and the difference in corrected $(F 435 W-F 625 W)$ color has a mean and standard deviation of 0.001 and 0.005 . This is encouraging in light of the fact that we have chosen the main sequence to construct the reddening map due to its greater number of stars (and hence spatial resolution) rather than the RGB, which is more nearly orthogonal to the reddening vector and thus should have even less sensitivity to $R_{V}$ (but at the cost of spatial resolution).

To illustrate the improvement in the photometry resulting from application of the reddening maps, CMDs of the ACS, WFPC2, and VVV photometry are shown before and after the application of these reddening corrections (using the reddening laws described in Section 6) in Figure 5.

\subsection{Observed CMD Features}

Several features are evident from the differential reddening corrected CMDs shown in the bottom row of Figure 5, as they sample a broad range of optical through near-infrared passbands. First, a blue HB is now discernible in all three corrected CMDs, consisting almost entirely of a tail extending at least to $F 625 W \sim 16.5(F 555 W \sim 17)$. In the $\left(K_{S}, J-K_{S}\right)$ plane, this blue tail is diagonal as is seen for other metal-poor GGCs in the near-infrared (e.g., Valenti et al. 2010; Chun et al. 2010), extending from $\left(J-K_{S}\right) \sim 0.6, K_{S} \sim 12$ to $\left(J-K_{S}\right) \sim 0.35$, $K_{S} \sim 14.5$. Second, the difference in line of sight reddening affecting the cluster versus the Galactic bulge, also noted by Valenti et al. (2010), is now clearly evident in the CMDs. While the bulge giant branch is largely absent from the shallower WFPC2 photometry, it is distinctly separated from the cluster RGBs in the ACS and VVV photometry. While the base of the cluster RGB occurs at $\left(J-K_{S}\right) \sim 0.85, K_{S} \sim 13.6$, the bulge red clump is visible at $\left(J-K_{S}\right) \sim 1.3, K_{S} \sim 13$ (nearinfrared fiducial sequences are presented in Section 3.4), with the bulge lower RGB intersecting the cluster main sequence around $\left(J-K_{S}\right) \sim 1.1, K_{S} \sim 17$. In the corrected ACS CMD in the lower left panel of Figure 5, the bulge main sequence is seen approximately parallel to the cluster main sequence and $\sim 0.5$ mag redder in $(F 435 W-F 625 W)$ color, and the bulge red clump stretches from $(F 435 W-F 625 W) \sim 4, F 625 W \sim 17.5$ to $(F 435 W-F 625 W) \sim 4.8, F 625 W \sim 19$, elongated along the reddening vector. Since differential reddening corrections were generated using the stellar population of NGC 6544, such an elongation seen after the application of these corrections implies that a large fraction of the reddening toward the Galactic 


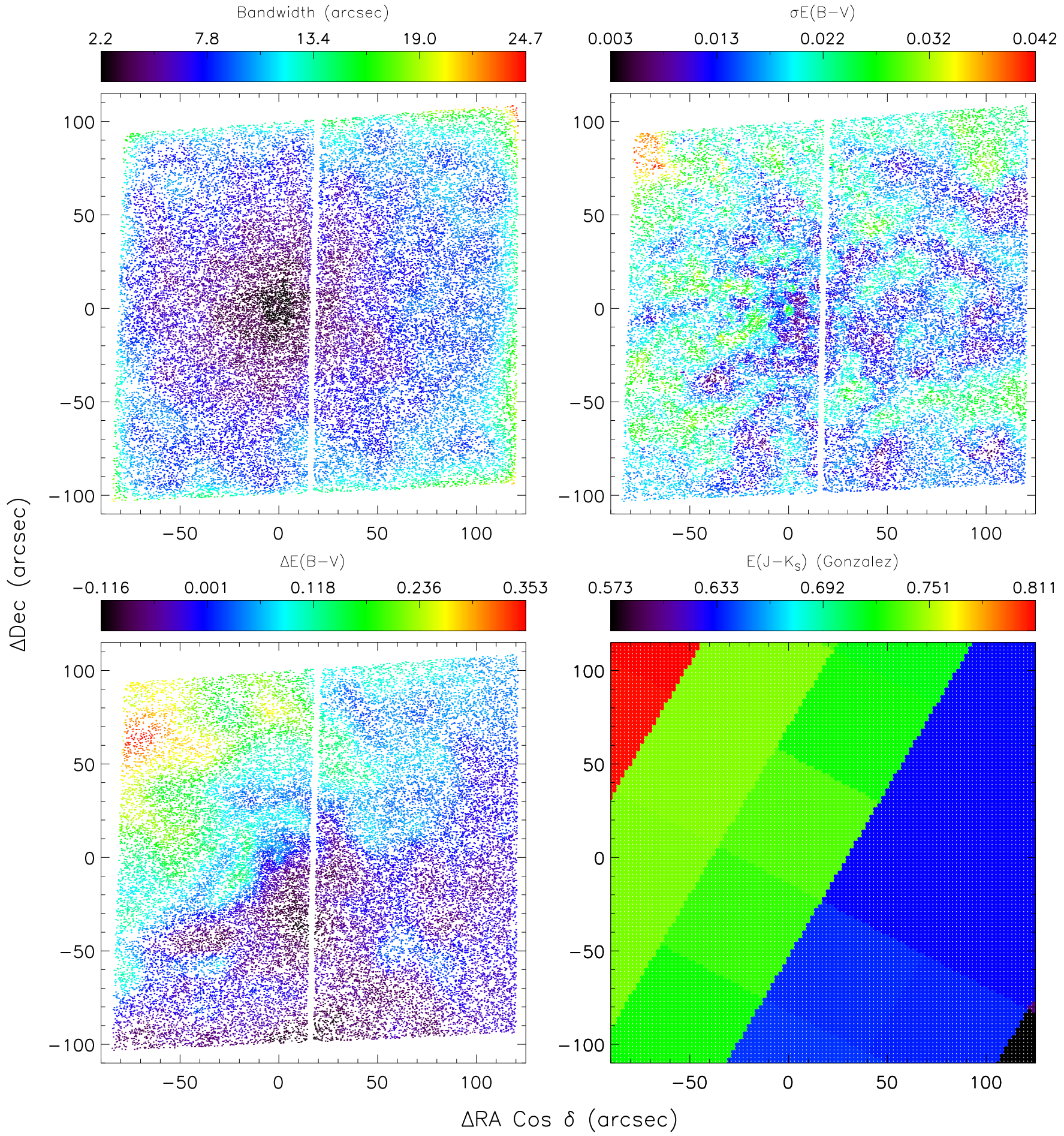

Figure 4. Maps from our dereddening procedure showing the final resulting spatial resolution (upper left), reddening correction uncertainty (upper right), and the final relative reddening corrections applied (lower left), as well as the reddening map of Gonzalez et al. (2012) for comparison (lower right). Positions are relative to the cluster center determined in Section 8.1.

bulge takes place behind the cluster (e.g., closer to the bulge) along the line of sight.

\subsection{A Proper-motion Selected Sample}

We explored the use of the WFPC 2 and ACS photometry to obtain relative proper motions, allowing us to quantify the extent to which the background population contaminates the region of the CMD coincident with the cluster evolutionary sequences. We selected a set of bright reference stars on the cluster RGB in common between both HST data sets, and attempted to isolate cluster stars via a simple iterative procedure. The offset between the WFPC2 and ACS photometry was measured using the reference stars as a positional zero point, eliminating stars with positional offsets more than $3 \sigma$ from the reference sample, and recentroiding until the number of stars in the reference sample converged to a constant value. This procedure was applied to each of the WFPC2 chips separately, and we found that while the lower spatial resolution of the WF chips prevented the reliable separation of cluster and field stars, a clear separation is evident 

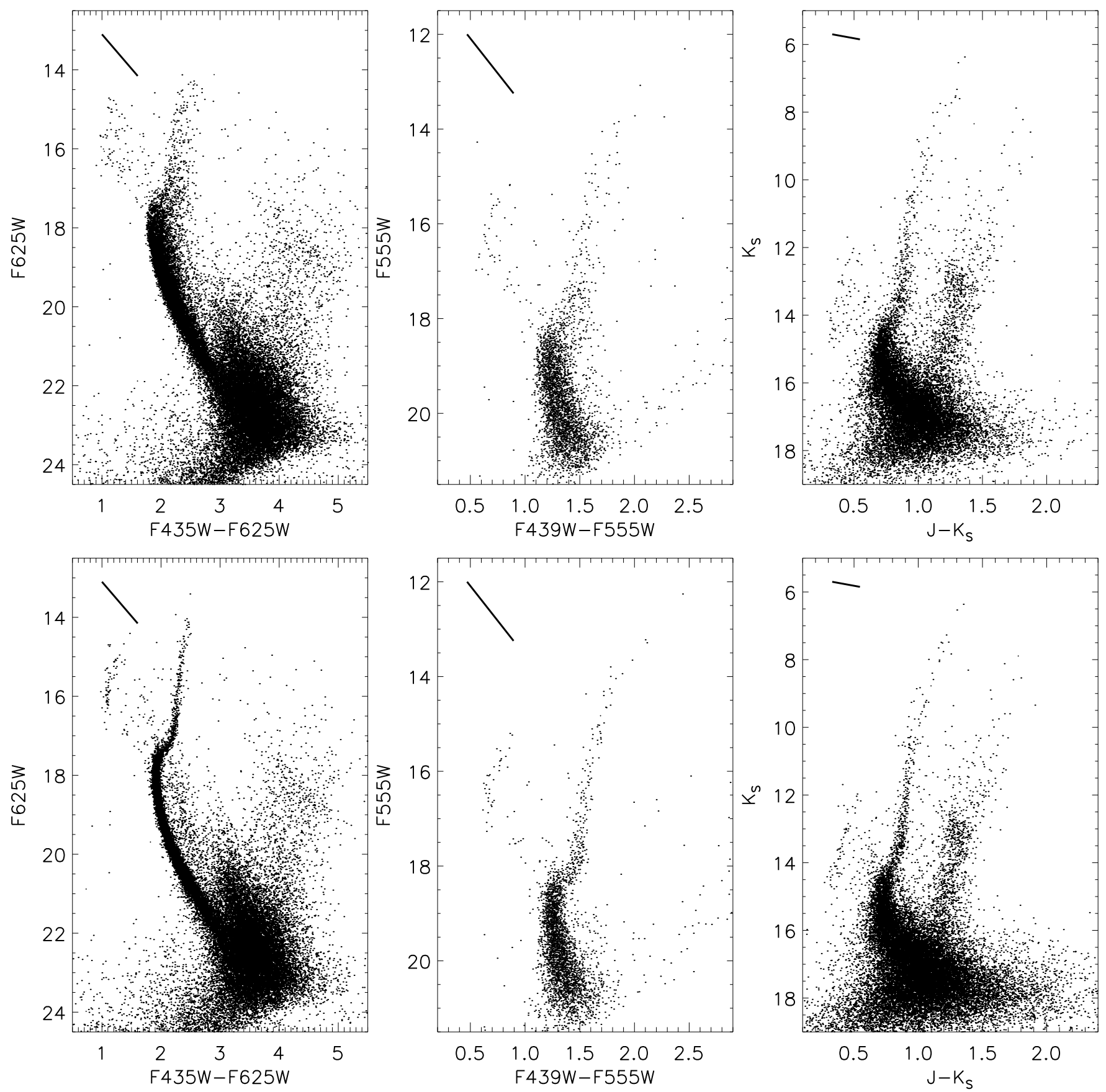

Figure 5. Color-magnitude diagrams of our photometry from ACS (left), WFPC2 (center), and VVV (right), shown before the application of the reddening map (top row) and afterward for comparison (bottom row). The reddening vector corresponding to $\Delta E(B-V)=0.4$ is shown as a thick line in the upper left corner of each CMD.

(Supplemental data of this figure are available in the online journal.)

in the case of the PC chip. There, we use the reference sample to define a cut to distinguish between cluster and field stars, shown in Figure 6. In actuality, proper-motion uncertainties should increase with magnitude, but we use a fixed (non-magnitude dependent) cut given the small available sample size, which is conservative in the sense that it will overestimate rather than underestimate the number of faint field stars that occupy the CMD region coincident with the cluster main sequence. The resulting proper-motion cleaned CMD shown in the left panel of Figure 6 reveals a population of centrally concentrated blue stragglers as well as evidence of a cluster binary sequence, although the small size of the PC chip field of view prevents a detailed quantitative analysis of these subpopulations for the time being.

\subsection{Near-infrared Fiducial Sequences From Cleaned CMDs}

While there has been a substantial effort toward characterizing GGC giant branches in the near-infrared (largely by Valenti et al. 2010, and references therein; also see Chun et al. 2010), there are only a handful of GGCs that currently have near-infrared photometric observations reaching down to, or below, the cluster main sequence turnoffs (Brasseur et al. 2010; Hendricks et al. 2012; Bono et al. 2010). For this reason, we provide fiducial 


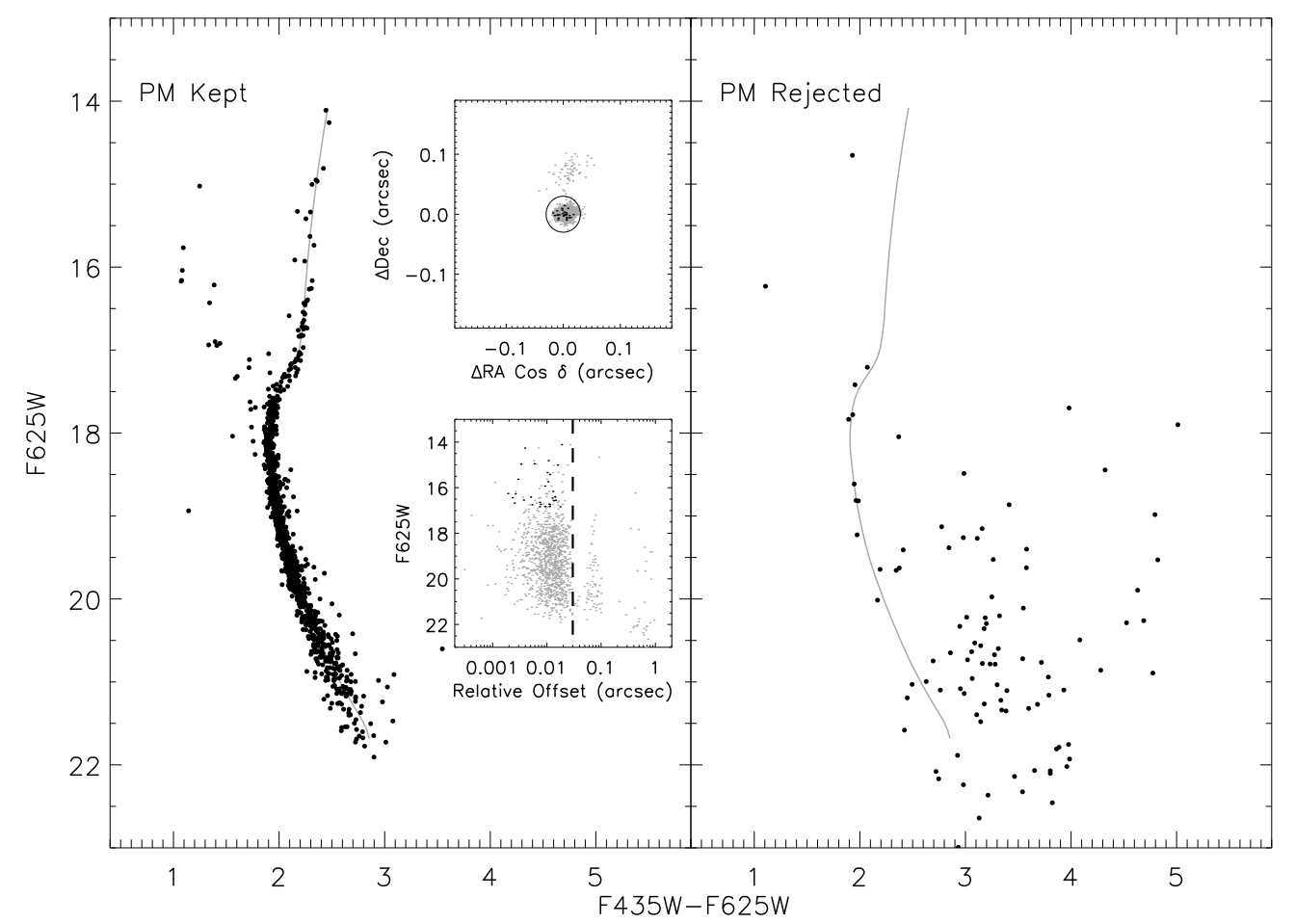

Figure 6. ACS CMDs showing the stars that passed (left) or failed (right) our proper-motion cut to select cluster members within the WFPC2 PC chip field of view. For reference, the fiducial sequence is shown as a gray line in both panels. The insets in the left-hand panel show the relative two-dimensional motions (top) with our cut illustrated as a black circle, and the total relative motion (bottom), with our cut indicated by the vertical dashed line. In both insets, the reference sample is shown as black rather than gray points.

sequences in the 2MASS $J H K_{S}$ system for NGC 6544 in case they may be of future use, especially in light of the relatively secure $[\mathrm{Fe} / \mathrm{H}]$ value for this cluster.

We statistically clean field stars from the CMD in order to characterize the cluster population to below the main sequence turnoff. Although in principle the bulge population could also be isolated from the cluster population using optical or optical-IR color-magnitude or color-color cuts, we found that a decontamination algorithm that employs the wide-field $J H K_{S}$ photometry (described below) was more effective at isolating the cluster main sequence in the near-infrared CMDs. Our proper-motion cuts from Section 3.3 are unfortunately insufficient for this purpose as the VVV photometry suffers from severe overcrowding in the central cluster region inside the WFPC2 PC chip field of view $\left(r \lesssim 20^{\prime \prime}\right)$.

Before proceeding with the decontamination algorithm, we must account for the fact that our comparison field can only be corrected for differential reddening using the Gonzalez et al. (2012) maps, which have a spatial resolution of more than an arcminute at best, whereas the cluster stars (those within the ACS field of view) have been corrected using our high spatial resolution map. This is important because the zero point of our (relative) map is arbitrary with respect to the Gonzalez et al. (2012) map, so we must ensure that the cluster and comparison field regions have been referred to the same reddening zero point before applying the decontamination procedure. To accomplish this, we compare the $\left(J-K_{S}\right)$ colors of the cluster stars after application of the Gonzalez et al. (2012) map to their colors after application of our high spatial resolution map. This comparison reveals that the zero point of our high resolution map corresponds to a median Gonzalez et al. (2012) map value of $E\left(J-K_{S}\right)=0.814$, with a standard deviation of 0.047 , reflecting the effect of reddening variations seen in our map that occur on a spatial scale too small for the Gonzalez et al. (2012) map to resolve.

The differential reddening corrected cluster CMDs are cleaned of field stars using a technique detailed in Bonatto \& Bica (2007), including recent improvements described by Bonatto \& Bica (2010). The decontamination procedure operates on a statistical basis, comparing relative stellar number densities in cluster and comparison fields within given boxes in color-magnitude space, fully taking observed photometric errors into account in a probabilistic manner. For convenience, we now summarize the operation of the cleaning procedure, using the nomenclature of Bonatto \& Bica (2007) for consistency.

1. We identify the spatial region to be decontaminated of field stars, which we refer to as the cluster region, with a projected spatial area denoted as $A_{\text {clus. }}$. In the present case, the cluster region is the ACS field of view. Next, we select a region representative of the field, which we refer to as the comparison region, with projected area $A_{\mathrm{fs}}$. Our comparison region has an inner radius from the cluster center of $r>19^{\prime}$ to ensure that it is effectively devoid of cluster members (see Section 8.2). The ratio of the comparison region area to the cluster region area $\left(A_{\mathrm{fs}} / A_{\text {clus }}\right)=32.8$.

2. Next, the CMDs of both the cluster region and comparison region are divided into three-dimensional grids in $J,(J-$ $\left.K_{S}\right),(J-H)$, as these planes yield the greatest variation among cluster CMD sequences as a function of cluster parameters (Bonatto et al. 2004). We have chosen the limits of these CMDs used in the decontamination procedure as $\left(-0.5<\left(J-K_{S}\right),(J-H)<2.5, J>5\right)$ so as to include all of the relevant evolutionary sequences seen in both the cluster and comparison regions. The faint limiting magnitude of the CMD region to be decontaminated, $J_{\text {lim }}$, 
Table 2

Field Star Decontamination Parameters

\begin{tabular}{lcccc}
\hline \hline $\begin{array}{l}J_{\lim } \\
(\mathrm{mag})\end{array}$ & $N$ & $N_{\text {comp }}$ & $\begin{array}{c}f_{\text {cl }} \\
(\%)\end{array}$ & $\begin{array}{c}\left\langle f_{\text {sub }}\right\rangle \\
(\%)\end{array}$ \\
\hline 18.84 & $24166 \pm 155$ & $692603 \pm 832$ & $32.2 \pm 3.1$ & $93.7 \pm 1.2$ \\
\hline
\end{tabular}

was set as the peak of the observed luminosity function (LF) in the cluster region. This is necessary since photometric incompleteness is a function of both magnitude and radius, so comparison fields are liable to contain faint stars whose analogs (bona fide field stars) lying within the cluster region will not be observable due to crowding. Within the aforementioned CMD limits, the three-dimensional cluster and comparison region CMDs are divided into boxes with sizes of $(0.5,0.1,0.1)$ in $\left(J, J-K_{S}, J-H\right)$, respectively, which we refer to as cells. We found these cell sizes to yield a good compromise between larger cells, which can degrade the observed evolutionary sequences in the CMD, and smaller cells, which fall victim to low subtraction efficiencies when too few stars are present within a given color-magnitude cell (see below). It is also worth noting that our chosen cell width in color is more than twice the value characterizing the small-scale reddening variations unaccounted for in the comparison map, mitigating the effects of the difference in spatial resolution between the reddening maps applied to the cluster and comparison regions.

3. Within each cell of the comparison region, the observed stellar number density $\rho_{\mathrm{fs}}$ is calculated taking photometric errors into account by summing the individual probability distributions of all stars in the cell before dividing by the comparison region area $A_{\mathrm{fs}}$ (see, e.g., Bonatto \& Bica 2007 for further details). The same operation is performed for all stars within the cluster region to obtain the observed (cluster+field) stellar number density in the cell, $\rho_{\text {obs }}^{\text {cell }}$. If $n_{\text {obs }}^{\text {cell }}$ represents the total number of stars in a cell in the cluster region, then the predicted number of field stars in this cell, based on comparison to the same CMD cell spatially located in the comparison region, is $n_{\mathrm{fs}}^{\text {cell }}=\left(\rho_{\mathrm{fs}}^{\text {cell }} / \rho_{\mathrm{obs}}^{\text {cell }}\right)$. The predicted number of bona fide cluster (non-field) stars within that cell is then $n_{\mathrm{cl}}^{\text {cell }}=n_{\mathrm{obs}}^{\text {cell }}-n_{\mathrm{fs}}^{\text {cell }}$.

4. The total number of predicted cluster stars within the cluster region, $N_{\mathrm{cl}}$, is then the sum of the values of $n_{\mathrm{cl}}^{\text {cell }}$ from each cell, summed over all cells. However, because we are working with number densities, the number of predicted field stars in each cell $n_{\mathrm{fs}}^{\text {cell }}$ is a decimal quantity, while in practice only an integer number of stars can be removed from the cell. For this reason, the field star subtraction efficiency $f_{\text {sub }}$ is defined as the ratio of the total (integer) number of field stars that were actually removed from the cluster region to the (decimal) number of predicted field stars in the cluster region, expressed as a percentage by multiplying by 100 , and serves as a gauge of the effectiveness of the cleaning procedure.

5. To mitigate the effects of using fixed three-dimensional CMD grids, steps $2-4$ are repeated, changing the CMD grid starting values by $\pm 1 / 3$ of the CMD grid size in each dimension, and also changing the grid size to 0.5 and 2 times the initial values in each dimension. In this way, the cleaning process in steps $2-4$ is repeated over a total of $729\left(=3^{6}\right)$ individual runs. If $\left\langle N_{\text {clus }}\right\rangle$ represents the average number of cluster stars that survived the cleaning process, averaged over all of the individual runs, then the final cleaned sample consists of the $\left\langle N_{\text {clus }}\right\rangle$ most probable cluster members within the cluster region. These most probable cluster members are obtained by sorting all stars in the cluster region by their survival frequency over all of the 729 individual runs, and keeping the $\left\langle N_{\text {clus }}\right\rangle$ stars with the highest survival frequencies. If $N$ represents the total number of stars in the cluster region, the fraction of cluster stars that survive the cleaning process, $f_{\mathrm{cl}}$, is then $\left\langle N_{\text {clus }}\right\rangle / N$.

The parameters most relevant to the decontamination process are summarized in Table 2, including the faint magnitude limit $J_{\text {lim }}$, the total number of stars in the cluster and comparison regions, $N$ and $N_{\text {comp }}$, the percentage of probable cluster stars surviving the cleaning procedure $f_{\mathrm{cl}}$, and the average field star subtraction efficiency $f_{\text {sub }}$. The results of the decontamination process are shown in Figure 7, and it is evident that the decontamination algorithm is particularly valuable to identify the cluster population faintward of the cluster subgiant branch, although a small fraction of bulge giants on the red side of the bulge RGB survive, likely due to small spatial scale reddening variations in the comparison field region unaccounted for by the Gonzalez et al. (2012) maps. The curvature at the faint end of the (cleaned) cluster main sequence in the $\left(K_{S}, J-K_{S}\right)$ plane in the left panel of Figure 7 likely results from the fact that the cleaning algorithm operates with the $J$ band on the magnitude axis of its (three-dimensional) CMD cells, and this is confirmed by the symmetry of the main sequence in the right-hand panel of Figure 7. However, in light of this feature, we note that we have additionally restricted our fiducial sequences in both CMDs of Figure 7 to terminate more than 1 mag brightward of $J_{\text {lim }}$. These fiducial sequences were calculated in both the $\left(K_{S}, J-K_{S}\right)$ and $(J, J-H)$ planes using only the $\left\langle N_{\text {clus }}\right\rangle$ probable cluster stars surviving the decontamination procedure, in the same manner as described in Section 3.1. We supply these sequences in Tables 3 and 4.

\section{CLUSTER AGE}

\subsection{Strategy for Measuring Age}

The distance- and reddening-independent diagnostics used to measure GGC ages from CMDs divide into those which are vertical and measure a magnitude difference (typically between the main sequence turnoff and the HB) and those which are horizontal and measure a color difference (see Sarajedini 2009 for a review of these methodologies). The vertical method has the advantage that it is less sensitive to variations in chemical abundances, but relies heavily on an accurate characterization of the HB magnitude, as discussed most recently by VandenBerg et al. 2013 (hereafter V13). For this reason, the ages of clusters with primarily blue HBs need to be measured by employing a horizontal rather than vertical method (e.g., Sarajedini \& Demarque 1990; Vandenberg et al. 1990). A potential pitfall of the horizontal method, explored by V13, is that it has a greater sensitivity to variations in chemical abundances, and is thus best employed in a relative sense via comparison to clusters (or empirically calibrated models) with the same abundances as the cluster under investigation. This complication can be mitigated to some extent by investigating relative ages using the prescription of Vandenberg et al. (1990), in which the fiducial sequence of each cluster is shifted in color to match their main sequence turnoff colors, and shifted in magnitude to match the fiducial sequence magnitudes at a point on the upper main 

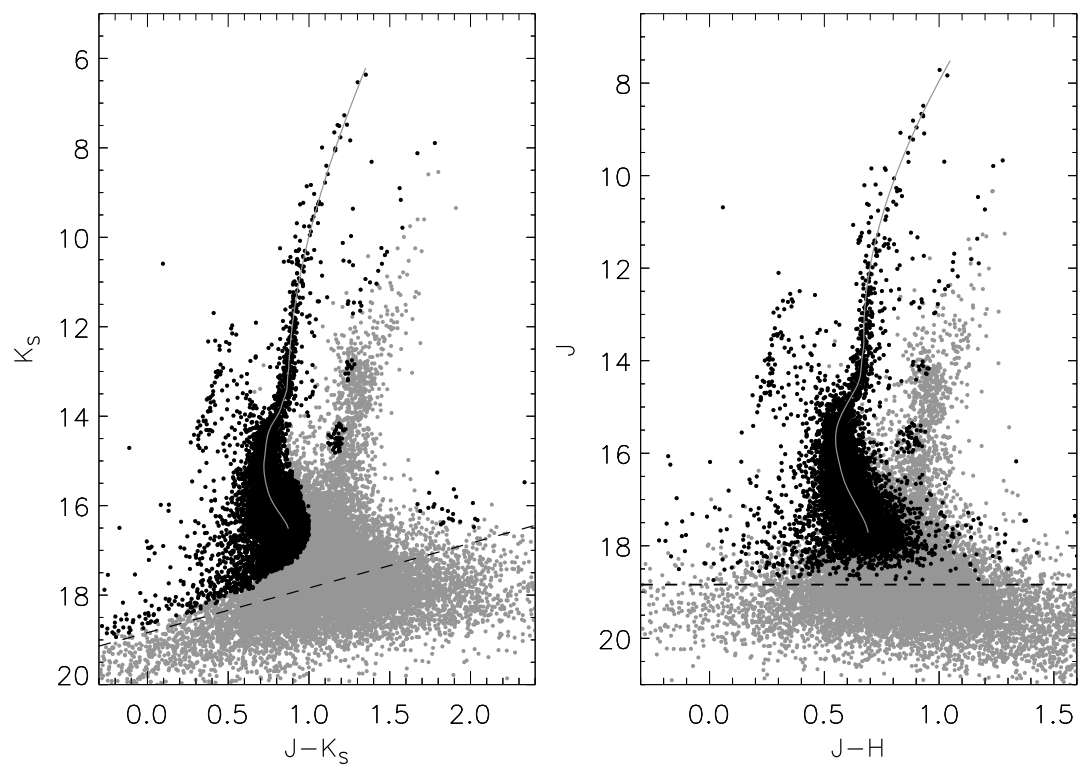

Figure 7. Left: differential-reddening corrected cluster CMD in the $\left(K_{S}, J-K_{S}\right)$ plane showing all stars in the ACS field of view in gray and those that survived the decontamination process in black. The dotted line indicates the faint magnitude limit $J_{\lim }$ for decontamination, and the cluster fiducial sequence is shown as a gray line. Right: same, but in the $(J, J-H)$ plane.

Table 3

NGC $6544\left(K_{S}, J-K_{S}\right)$ Fiducial Sequence

\begin{tabular}{lccccc}
\hline \hline$\left(J-K_{S}\right)$ & $K_{S}$ & $\left(J-K_{S}\right)$ & $K_{S}$ & $\left(J-K_{S}\right)$ & $K_{S}$ \\
\hline 1.3508 & 6.2137 & 1.0188 & 9.7137 & 0.8666 & 13.2137 \\
1.3397 & 6.3137 & 1.0115 & 9.8137 & 0.8653 & 13.3137 \\
1.3286 & 6.4137 & 1.0044 & 9.9137 & 0.8622 & 13.4137 \\
1.3177 & 6.5137 & 0.9975 & 10.0137 & 0.8539 & 13.5137 \\
1.3068 & 6.6137 & 0.9907 & 10.1137 & 0.8441 & 13.6137 \\
1.2959 & 6.7137 & 0.9841 & 10.2137 & 0.8343 & 13.7137 \\
1.2852 & 6.8137 & 0.9776 & 10.3137 & 0.8264 & 13.8137 \\
1.2745 & 6.9137 & 0.9713 & 10.4137 & 0.8152 & 13.9137 \\
1.2640 & 7.0137 & 0.9652 & 10.5137 & 0.8001 & 14.0137 \\
1.2535 & 7.1137 & 0.9593 & 10.6137 & 0.7825 & 14.1137 \\
1.2431 & 7.2137 & 0.9535 & 10.7137 & 0.7662 & 14.2137 \\
1.2327 & 7.3137 & 0.9479 & 10.8137 & 0.7541 & 14.3137 \\
1.2225 & 7.4137 & 0.9425 & 10.9137 & 0.7449 & 14.4137 \\
1.2124 & 7.5137 & 0.9372 & 11.0137 & 0.7392 & 14.5137 \\
1.2024 & 7.6137 & 0.9322 & 11.1137 & 0.7348 & 14.6137 \\
1.1924 & 7.7137 & 0.9273 & 11.2137 & 0.7319 & 14.7137 \\
1.1826 & 7.8137 & 0.9226 & 11.3137 & 0.7287 & 14.8137 \\
1.1729 & 7.9137 & 0.9181 & 11.4137 & 0.7258 & 14.9137 \\
1.1633 & 8.0137 & 0.9138 & 11.5137 & 0.7220 & 15.0137 \\
1.1538 & 8.1137 & 0.9097 & 11.6137 & 0.7208 & 15.1137 \\
1.1444 & 8.2137 & 0.9058 & 11.7137 & 0.7215 & 15.2137 \\
1.1351 & 8.3137 & 0.9022 & 11.8137 & 0.7242 & 15.3137 \\
1.1260 & 8.4137 & 0.8987 & 11.9137 & 0.7287 & 15.4137 \\
1.1169 & 8.5137 & 0.8954 & 12.0137 & 0.7342 & 15.5137 \\
1.1080 & 8.6137 & 0.8923 & 12.1137 & 0.7412 & 15.6137 \\
1.0992 & 8.7137 & 0.8894 & 12.2137 & 0.7485 & 15.7137 \\
1.0906 & 8.8137 & 0.8868 & 12.3137 & 0.7593 & 15.8137 \\
1.0820 & 8.9137 & 0.8843 & 12.4137 & 0.7728 & 15.9137 \\
1.0736 & 9.0137 & 0.8821 & 12.5137 & 0.7888 & 16.0137 \\
1.0654 & 9.1137 & 0.8801 & 12.6137 & 0.8064 & 16.1137 \\
1.0572 & 9.2137 & 0.8772 & 12.7137 & 0.8260 & 16.2137 \\
1.0493 & 9.3137 & 0.8755 & 12.8137 & 0.8444 & 16.3137 \\
1.0414 & 9.4137 & 0.8752 & 12.9137 & 0.8614 & 16.4137 \\
1.0337 & 9.5137 & 0.8721 & 13.0137 & 0.8724 & 16.5137 \\
1.0262 & 9.6137 & 0.8682 & 13.1137 & & \\
\hline & & & & & \\
& & & & \\
& & & & \\
& & & &
\end{tabular}

Table 4

NGC $6544(J, J-H)$ Fiducial Sequence

\begin{tabular}{lccccc}
\hline \hline$(J-H)$ & $J$ & $(J-H)$ & $J$ & $(J-H)$ & $J$ \\
\hline 1.0481 & 7.5155 & 0.7438 & 11.0155 & 0.6453 & 14.5155 \\
1.0369 & 7.6155 & 0.7382 & 11.1155 & 0.6349 & 14.6155 \\
1.0258 & 7.7155 & 0.7329 & 11.2155 & 0.6238 & 14.7155 \\
1.0149 & 7.8155 & 0.7277 & 11.3155 & 0.6125 & 14.8155 \\
1.0041 & 7.9155 & 0.7228 & 11.4155 & 0.6011 & 14.9155 \\
0.9934 & 8.0155 & 0.7181 & 11.5155 & 0.5901 & 15.0155 \\
0.9828 & 8.1155 & 0.7136 & 11.6155 & 0.5796 & 15.1155 \\
0.9724 & 8.2155 & 0.7093 & 11.7155 & 0.5703 & 15.2155 \\
0.9620 & 8.3155 & 0.7053 & 11.8155 & 0.5621 & 15.3155 \\
0.9519 & 8.4155 & 0.7014 & 11.9155 & 0.5558 & 15.4155 \\
0.9419 & 8.5155 & 0.6978 & 12.0155 & 0.5520 & 15.5155 \\
0.9320 & 8.6155 & 0.6944 & 12.1155 & 0.5496 & 15.6155 \\
0.9222 & 8.7155 & 0.6913 & 12.2155 & 0.5492 & 15.7155 \\
0.9127 & 8.8155 & 0.6883 & 12.3155 & 0.5499 & 15.8155 \\
0.9032 & 8.9155 & 0.6856 & 12.4155 & 0.5524 & 15.9155 \\
0.8939 & 9.0155 & 0.6832 & 12.5155 & 0.5561 & 16.0155 \\
0.8848 & 9.1155 & 0.6809 & 12.6155 & 0.5605 & 16.1155 \\
0.8758 & 9.2155 & 0.6790 & 12.7155 & 0.5655 & 16.2155 \\
0.8670 & 9.3155 & 0.6772 & 12.8155 & 0.5699 & 16.3155 \\
0.8583 & 9.4155 & 0.6757 & 12.9155 & 0.5751 & 16.4155 \\
0.8498 & 9.5155 & 0.6745 & 13.0155 & 0.5810 & 16.5155 \\
0.8415 & 9.6155 & 0.6735 & 13.1155 & 0.5876 & 16.6155 \\
0.8334 & 9.7155 & 0.6727 & 13.2155 & 0.5963 & 16.7155 \\
0.8254 & 9.8155 & 0.6722 & 13.3155 & 0.6056 & 16.8155 \\
0.8176 & 9.9155 & 0.6720 & 13.4155 & 0.6157 & 16.9155 \\
0.8099 & 10.0155 & 0.6720 & 13.5155 & 0.6264 & 17.0155 \\
0.8025 & 10.1155 & 0.6723 & 13.6155 & 0.6371 & 17.1155 \\
0.7952 & 10.2155 & 0.6709 & 13.7155 & 0.6484 & 17.2155 \\
0.7881 & 10.3155 & 0.6681 & 13.8155 & 0.6591 & 17.3155 \\
0.7812 & 10.4155 & 0.6650 & 13.9155 & 0.6696 & 17.4155 \\
0.7745 & 10.5155 & 0.6630 & 14.0155 & 0.6791 & 17.5155 \\
0.7679 & 10.6155 & 0.6612 & 14.1155 & 0.6863 & 17.6155 \\
0.7616 & 10.7155 & 0.6593 & 14.2155 & 0.6911 & 17.7155 \\
\hline & & & & &
\end{tabular}


Table 5

Reference Cluster Parameters

\begin{tabular}{|c|c|c|c|}
\hline$(m-M)_{0}$ & $E(B-V)$ & Age (Gyr) & Reference \\
\hline \multicolumn{4}{|c|}{$\mathrm{M} 3[\mathrm{Fe} / \mathrm{H}]_{C 09}=-1.50 \pm 0.05$} \\
\hline 15.05 & 0.018 & $12.50 \pm 0.50$ & Dotter et al. (2010) \\
\hline 14.98 & 0.013 & $11.75 \pm 0.25$ & V13 \\
\hline \multicolumn{4}{|c|}{$\mathrm{M} 72[\mathrm{Fe} / \mathrm{H}]_{C 09}=-1.48 \pm 0.07$} \\
\hline 16.11 & 0.049 & $12.75 \pm 0.75$ & Dotter et al. (2010) \\
\hline 16.15 & 0.05 & & Recio Blanco et al. (2005) \\
\hline 16.06 & 0.057 & $11.50 \pm 0.25$ & V13 \\
\hline \multicolumn{4}{|c|}{$\mathrm{M} 5[\mathrm{Fe} / \mathrm{H}]_{C 09}=-1.33 \pm 0.02$} \\
\hline 14.32 & 0.032 & $12.25 \pm 0.75$ & Dotter et al. (2010) \\
\hline 14.37 & 0.03 & & Recio Blanco et al. (2005) \\
\hline 14.26 & 0.038 & $11.50 \pm 0.25$ & V13 \\
\hline \multicolumn{4}{|c|}{$\mathrm{M} 13[\mathrm{Fe} / \mathrm{H}]_{C 09}=-1.58 \pm 0.04$} \\
\hline 14.44 & 0.019 & $13.00 \pm 0.50$ & Dotter et al. (2010) \\
\hline 14.42 & 0.02 & & Recio Blanco et al. (2005) \\
\hline 14.40 & 0.017 & $12.00 \pm 0.38$ & V13 \\
\hline \multicolumn{4}{|c|}{$\mathrm{NGC} 1851[\mathrm{Fe} / \mathrm{H}]_{C 09}=-1.18 \pm 0.04$} \\
\hline 15.41 & 0.02 & & Recio Blanco et al. (2005) \\
\hline 15.33 & 0.034 & $11.00 \pm 0.25$ & V13 \\
\hline
\end{tabular}

sequence 0.05 mag redder in color than the turnoff. ${ }^{8}$ A difference in age is then immediately apparent as a difference in color of the cluster RGBs at a fixed reference magnitude (following V13, we adopt the magnitude 2.8 mag brighter than the turnoff). This methodology circumvents two common pitfalls associated with determining GGC ages: first, V13 demonstrate that if the age is ascertained solely by finding the isochrone that best fits the observed cluster sequence, there is a significant degeneracy when using the isochrone fits to the subgiant branch to determine the cluster age while also allowing the distance and reddening to vary. Secondly, and relatedly, the CMD morphology of GGC turnoff regions is nearly independent of age and metallicity, especially at $[\mathrm{Fe} / \mathrm{H}] \lesssim-1.4$ (see Figure 2 of $\mathrm{V} 13$ ). We refer the reader to Sections 3.1 and 3.2 of V13 for a more detailed discussion of these issues, but emphasize that the registration of cluster fiducial sequences following the Vandenberg et al. (1990) prescription is employed here in a strictly empirical sense. Our strategy is to use clusters with similar spectroscopic metallicities to NGC 6544 as well as homogeneously determined absolute ages from V13, thereby ascertaining the age of NGC 6544 via a relative comparison of the cluster fiducial sequences following the Vandenberg et al. (1990) prescription.

We wish to exploit the relative precision of the ACS and WFPC2 photometry to investigate the age of NGC 6544 , and have therefore processed available archival HST WFPC2 and ACS WFC imaging of comparison clusters with similar metallicities so that fiducial sequences of these comparison clusters can be generated. The WFPC 2 and ACS imaging employed for this purpose is listed in Table 1, and has been processed using HSTPHOT and DOLPHOT identically as for NGC 6544, with fiducial sequences generated in the same manner as described in Section 3.1. In Table 5, we list all of the GGCs that we employ for empirical comparisons to NGC 6544, along with their spectroscopic $[\mathrm{Fe} / \mathrm{H}]$ from Carretta et al. (2009) and their

\footnotetext{
8 Although V13 use the value of 0.05 in ACS WFC $F 606 W-F 814 W$ color, we employ it here for the WFPC2 $F 439 W-F 555 W$ and ACS $F 435-F 625$ color since V13 demonstrated that this value is robust and can be employed in $B-V$ or $V-I$ colors as well (see their Figure 6).
}

ages, distances, and reddenings as determined from several recent large-scale space-based GGC imaging studies. Since the Vandenberg et al. (1990) technique for relative age measurement is distance- and reddening-independent, the comparison cluster distances and reddenings come into play only later in Section 5.1, where we use this ensemble of photometry to estimate the distance and reddening of NGC 6544.

\subsection{Age from WFPC2 Fiducial Sequences}

The two GGCs M3 and M72 have spectroscopic metallicities identical to that of NGC 6544 to within their uncertainties, and are thus ideal for an empirical comparison. Although neither of these clusters has available photometry in the ACS WFC filter pair used to observed NGC 6544 (or $B, R$ photometry which could be transformed to these filters in light of their low reddening), we have reduced WFPC2 $F 439 \mathrm{~W}, F 555 \mathrm{~W}$ photometry of M72, and in order to obtain fiducial sequences for M3, we obtained $B, V$ broadband photometry from Peter Stetson's diligently maintained and calibrated CADC archive of photometric standard fields ${ }^{9}$ and converted this photometry to the WFPC2 $F 439 W, F 555 W$ plane using the latest calibrations kindly made publicly available by A. Dolphin. ${ }^{10}$ The WFPC2 photometry of M3 and M72 is shown in Figure 8, and the result of applying the Vandenberg et al. (1990) registration prescription is shown in Figure 9, suggesting that NGC 6544 is slightly older than M3 and M72. The quality of the photometry is the limiting factor in this determination, and this is evidenced by the reversal of the relative RGB locations of M3 and M72 as compared to their age measurements from V13. To further quantify this observational uncertainty, we have performed a set of simulations wherein for each iteration, all stars are randomly shifted in the CMD by a random amount drawn from a Gaussian distribution with a standard deviation equal to the photometric error at the magnitude of that star (ascertained from the artificial star tests). For each of 1000 such iterations, a fiducial sequence is fit in an identical manner as for the observed photometry (using the method in Section 3.1), and the color along the fiducial sequence at the reference magnitude on the RGB is reported (where this magnitude is defined based on the measured turnoff color following the Vandenberg prescription). We have performed such an ensemble of simulations for each of the three clusters shown in Figure 8, and we find that the differences in the fiducial sequence color at the reference magnitude are only marginally significant. Specifically, we measure the fiducial sequence color difference $\Delta(F 439 \mathrm{~W}-$ $F 555 W)-(F 439 W-F 555 W)_{\mathrm{TO}}=0.028 \pm 0.011$ and $-0.014 \pm 0.012$ for M3 and M72, respectively (in the sense NGC 6544 fiducial color minus reference cluster fiducial color) at the reference magnitude 2.8 mag brighter than the observed main sequence turnoff of NGC 6544. Furthermore, we have not taken into account the additional uncertainty in the photometric transformation from the ground-based Stetson $B, V$ magnitudes for M3 to the WFPC2 ( $F 439 W, F 555 W)$ system, ${ }^{11}$ so we must conclude that we cannot usefully constrain the age difference between NGC 6544 and the other two clusters using the WFPC2 photometry alone. We therefore look to the deeper ACS photometry for a second opinion.

\footnotetext{
9 http://www1.cadc-ccda.hia-iha.nrc-cnrc.gc.ca/community/STETSON/

$10 \mathrm{http}: / /$ purcell.as.arizona.edu/wfpc2_calib

11 The latest calibrations provided by A. Dolphin give photometric zero point uncertainties of $<0.01 \mathrm{mag}$ but do not give uncertainties on the color term. The original calibrations from Holtzman et al. (1995, see, e.g., their Figures 4 and 9) imply uncertainties on the order of $\sim 0.01 \mathrm{mag}$.
} 


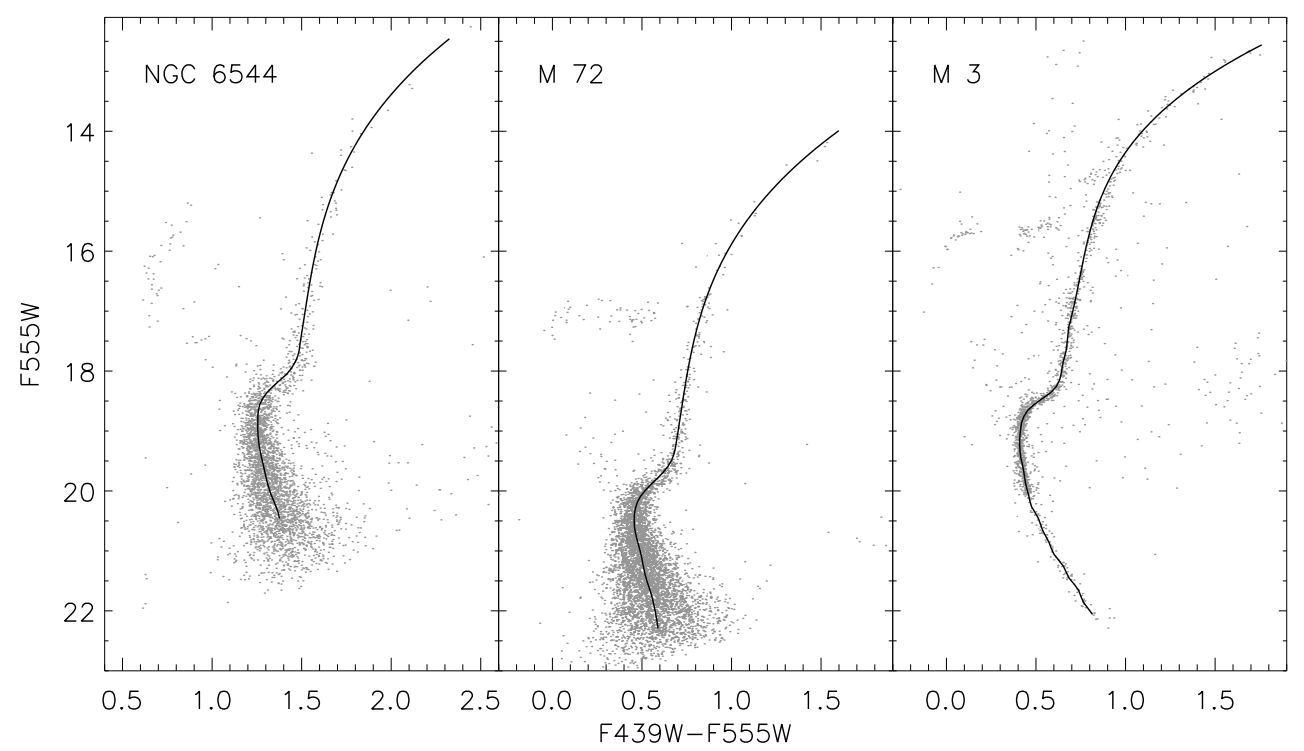

Figure 8. WFPC2 photometry for NGC 6544 (left), M72 (center), and M3 (right). The NGC 6544 photometry is corrected for differential reddening, and the M3 photometry is that of Peter Stetson's $B, V$ standards, transformed to the WFPC2 filter system. The derived fiducial sequences are overplotted in black.

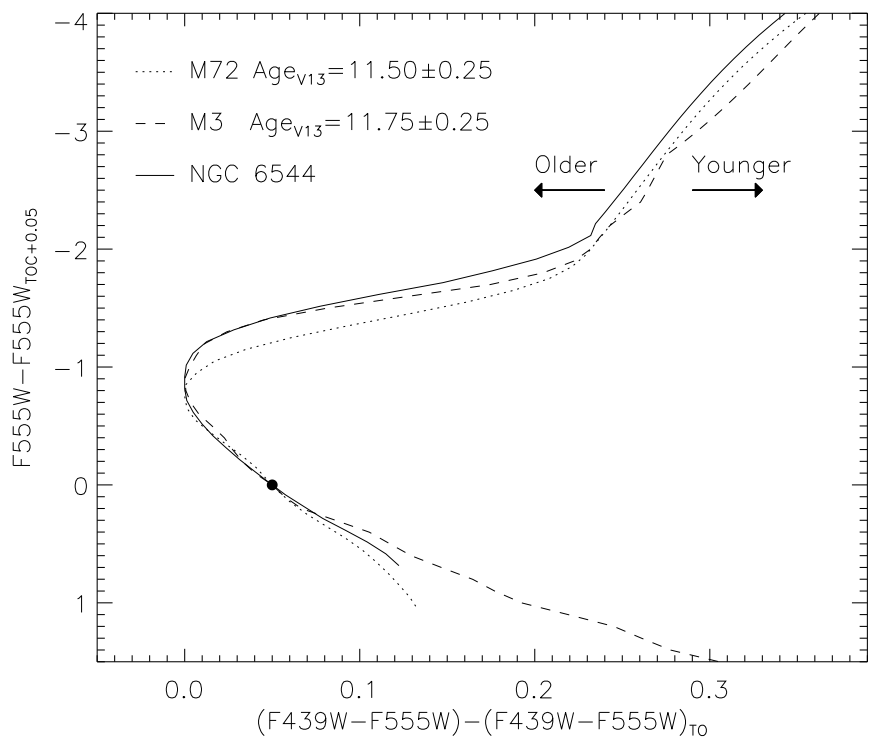

Figure 9. WFPC2 $F 439 W-F 555 W$ fiducial sequences of NGC 6544 (solid line), M3 (dashed), and M72 (dotted), registered following the prescription of Vandenberg et al. (1990). The sequences were registered horizontally to their turnoff colors, and the point on the main sequence 0.05 redder than this color, used to register the sequences vertically, is shown as a filled circle. The ages for M3 and M72 measured by V13 using the vertical method are given (in Gyr), and the arrows indicate the offset in giant branch color corresponding to a positive or negative change in age.

\subsection{Age from ACS Fiducial Sequences}

There are unfortunately no nearby comparison clusters that have both metallicities identical to NGC 6544 as well as available photometry in suitable filters for comparison to our ACS $(F 435 W, F 625 W)$ data, so we have chosen two clusters bracketing its observed $[\mathrm{Fe} / \mathrm{H}], \mathrm{M} 5$ and M13. As the ACS photometry for these clusters saturates below the HB, we have supplemented the ACS photometry of M5 with photometry from the CADC archive, which was converted to $(F 435 W, F 625 W)$ using coefficients from Sirianni et al. (2005) and employed for $F 625 W<17.1$. The $(F 435 W, F 625 W)$ photometry of M5 and M13 and the resultant fiducial sequences are shown in Figure 10. Unlike the case of the WFPC2 photometry, we must account for the fact that the GGCs being compared have (slightly) different metallicities when registering the cluster fiducial sequences. Following V13, we derive a color correction to be applied to the RGB color of each cluster via comparison with models. We measure the color corrections to be applied in the cases of M5 and M13 at the chosen reference magnitude 2.8 mag brighter than the turnoff (corresponding to $F 625 \mathrm{~W}-$ $F 625 W_{\text {TOC }+0.05}=-3.5$ ) using isochrones from the Dartmouth Stellar Evolution Database (DSED; Dotter et al. 2008). A grid of DSED isochrones at the metallicity of NGC $6544([\mathrm{Fe} / \mathrm{H}]=$ $-1.50,[\alpha / \mathrm{Fe}]=0.4$, corresponding to $[\mathrm{M} / \mathrm{H}]=-1.21$, the spectroscopically observed value) with ages from 10-14 Gyr, in intervals of $1 \mathrm{Gyr}$, is compared to a similar grid at the Dotter et al. (2010) metallicity of each of the comparison clusters, and the color difference at the RGB reference magnitude is measured after the isochrones have all been registered following the Vandenberg et al. (1990) prescription. As V13 discuss, this process does not require that the isochrones correctly reproduce observed colors for all the evolutionary sequences, and instead is reliant on the isochrones only to obtain the difference in the RGB color shift as a function of metallicity. We illustrate this measurement for M5 and M13 in the right-hand panels of Figure 10, which is zoomed in on the RGB in the color and magnitude range of the RGB reference magnitude. In the top two panels, registered isochrones at the metallicities of M5 (top) and M13 (middle) are compared to those of NGC 6544 to measure the mean color correction at the RGB reference magnitude. In the bottom panel, the registered fiducial sequences for the three clusters are plotted after application of the color correction, and the value of the correction is illustrated as a short horizontal line (drawn at the RGB reference magnitude) connected to a portion of the original, uncorrected fiducial sequence.

The implication from the bottom right-hand panel of Figure 10 is that NGC 6544 is older than M5, and may be slightly older than M13. However, to quantify the total uncertainty on the (registered) fiducial sequence colors at the RGB reference magnitude, we take several sources of error into account and quantify this total color uncertainty as

$$
\sigma_{\mathrm{col}}^{2}=\sigma_{\mathrm{obs}}^{2}+\sigma_{\mathrm{CC}, \text { age }}^{2}+\sigma_{\mathrm{CC},[\mathrm{Fe} / \mathrm{H}]}^{2}
$$



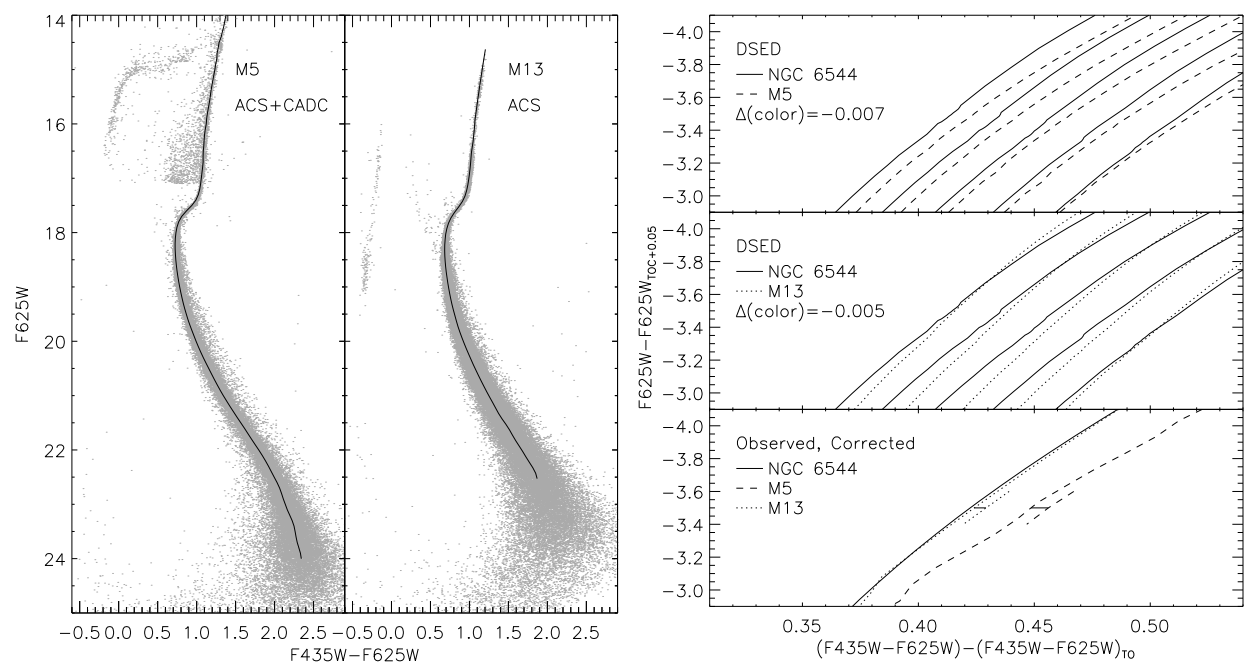

Figure 10. ACS WFC photometry for M5 (left) and M13 (center), with fiducial sequences overplotted. For M5, CADC $B, R$ photometry was employed for $F 625 W<17.1$, converted to the ACS WFC system using coefficients from Sirianni et al. (2005). Right: comparison between 10-14 Gyr DSED isochrones at the metallicity of NGC 6544 vs. those at the metallicity of each comparison cluster, registered using the Vandenberg et al. (1990) prescription. The color correction is measured 2.8 mag above the main sequence turnoff, at $F 625-F 625 \mathrm{TOC}+0.05=-3.5$. In the bottom right panel, we show the fiducial sequences of all three clusters after application of the color corrections to the comparison clusters. The value of the color correction for each comparison cluster is given in the upper plots, and illustrated in the bottom plot with a horizontal line connected to an uncorrected portion of the fiducial sequence, with the horizontal line located at a magnitude of $F 625-F 625_{\mathrm{TOC}+0.05}=-3.5$.

Here, $\sigma_{\text {obs }}$ represents the observational uncertainty in the fiducial sequence color due to photometric errors, which we have evaluated using simulations in the same manner as for the WFPC2 photometry above. The remaining two terms involve uncertainties in the color corrections predicted from the DSED models: $\sigma_{\mathrm{CC} \text {,age }}$ is the standard deviation of the mean color correction derived from DSED isochrones ranging from $10-14$ Gyr in age. Meanwhile, $\sigma_{\mathrm{CC},[\mathrm{Fe} / \mathrm{H}]}$ is the uncertainty in the color correction resulting from the uncertainties in the cluster $[\mathrm{Fe} / \mathrm{H}]$ values listed in Carretta et al. (2009), which we denote as $\sigma[\mathrm{Fe} / \mathrm{H}]$. These values were ascertained by rederiving the color correction using the DSED isochrones, but varying the metallicity of isochrones corresponding to NGC 6544 and each reference cluster by $\pm \sigma[\mathrm{Fe} / \mathrm{H}]$. This last term, specifically the larger uncertainty listed for the metallicity of NGC 6544, turns out to be the dominant source of uncertainty in our age determinations when calculating the total fiducial sequence color at the reference magnitude $\sigma_{\text {col }}$. Taking into account the photometric uncertainty in the fiducial sequence color of NGC 6544, we measure color differences at the reference magnitude of $\Delta(F 435 W-F 625 W)-(F 435 W-F 625 W)_{\mathrm{TO}}=-0.026 \pm$ 0.019 and $-0.004 \pm 0.016$ compared to M5 and M13 respectively, where the given uncertainties represent the total $\sigma_{\mathrm{col}}$. Therefore, we find that NGC 6544 is coeval with M13 to well within the relevant uncertainties. Regarding M5, although the color difference at the reference point appears marginally significant, we have neglected the uncertainty in the transformation from the CADC $B, R$ filters to the ACS filters in the calculation of $\sigma_{\text {col }}$. The uncertainties in the transformation coefficients of Sirianni et al. (2005) imply that these are on the order of a few percent, but additionally V13 and Dotter et al. (2010) both list absolute ages for M5 and M13 which are coeval to within their uncertainties. We therefore abstain from placing more quantitative limits on the age of NGC 6544.

\section{DISTANCE AND REDDENING}

We choose to estimate the distance and extinction to NGC 6544 via direct comparison to photometry of other GGCs rather than relying completely on evolutionary models. This choice is based on the fact that current models can show offsets in optical colors (e.g., Dotter et al. 2008; V13) as well as optical-infrared and infrared colors (Brasseur et al. 2010). For this reason, it is crucial that these models be tested thoroughly before being applied, and in our case such broad tests have not been undertaken for the bandpasses and metallicity range of NGC 6544. Therefore, we estimate the values of $(m-M)_{0}$ and $E(B-V)$ for NGC 6544 in an entirely empirical fashion, by comparing our photometry in various color-magnitude planes to publicly available photometry for clusters which have low reddenings and similar spectroscopic metallicities. In order to perform this comparison, we use the reddening law of Hendricks et al. (2012), interpolating in the tables in their Appendix B to obtain the extinction coefficients for $\mathrm{VIJHK}_{S}$ filters (noting that the Rosenberg et al. 2000 photometry is calibrated to Landolt 1992 standards). We assume a standard reddening law, and this assumption as well as the extinction coefficients employed for the HST filters are discussed in Section 6.

\subsection{Empirical Comparison to Reference Clusters}

The reddening of NGC 6544 is somewhat more easily constrained than its distance, which is difficult to determine even in a relative sense because of the sparse, blue nature of the cluster HB. Our strategy is to adjust the distance and reddening of NGC 6544 to obtain the best match to the available comparison cluster photometry in each of several CMD planes independently, choosing comparison clusters with metallicities that are either identical to that of NGC 6544 to within their uncertainties, or if unavailable, choosing clusters with metallicities bracketing that of NGC 6544. In order to compare our ensemble of comparison cluster photometry in various bandpasses directly to that of NGC 6544, we must assume distances and reddenings for the comparison clusters. Fortunately, because we have selected well-studied comparison clusters that have relatively low reddenings, recent studies are in fairly good agreement on their distance and reddening values (see Table 5). Hence, for consistency we adopt the distances and reddenings listed by V13 since this is the only study that gives homogeneously obtained distance moduli and reddenings 
Table 6

NGC 6544 Distance and Reddening Estimates

\begin{tabular}{lcr}
\hline \hline$\Lambda_{1}-\Lambda_{2}, M$ & $E\left(\Lambda_{1}-\Lambda_{2}\right)$ & $(m-M)_{0}$ \\
\hline$V-K_{S}, V$ & $2.21 \pm 0.03$ & $11.95 \pm 0.2$ \\
$V-J, V$ & $1.90 \pm 0.03$ & $11.5 \pm 0.2$ \\
$J-K_{S}, K_{S}$ & $0.43 \pm 0.03$ & $11.95 \pm 0.2$ \\
$F 435 W-F 625 W, F 625 W$ & $1.25 \pm 0.03$ & $12.05 \pm 0.1$ \\
$F 439 W-F 555 W, F 555 W$ & $0.87 \pm 0.03$ & $12.05 \pm 0.2$ \\
$V-I, V$ & $1.01 \pm 0.05$ & $12.1 \pm 0.3$ \\
\hline
\end{tabular}

for all of our comparison clusters, although the impact of this choice is discussed below in Section 5.4.1.

We obtain an independent estimate of the distance and reddening from each color-magnitude plane by shifting our NGC 6544 photometry vertically and horizontally to match the relevant reference cluster photometry. In order to keep this analysis independent of cluster age, the region of the main sequence turnoff and subgiant branch are not considered when undertaking this comparison. In each color-magnitude plane, the optimal vertical and horizontal shifts yield a distance and reddening value for NGC 6544, and these individual values are listed for each color-magnitude plane (with magnitude $M$ on the $y$-axis and color $\Lambda_{1}-\Lambda_{2}$ on the $x$-axis) in Table 6 . The best-fit values listed have been determined visually, and we have attempted to conservatively estimate their uncertainties as the offset from the best-fit values which would render the NGC 6544 and comparison cluster sequences incompatible beyond their observed width. Although this is admittedly a somewhat subjective approach, the various sources of comparison cluster photometry and their uncertainties (for example, the availability of artificial star tests) are quite heterogeneous in terms of photometric quality and the evolutionary sequences available for comparison. This is illustrated in Figure 11, where we plot the photometry of NGC 6544 in black, along with the comparison cluster photometry, which is color-coded by cluster, shifted using the weighted mean of the values listed in Table 6 obtained from each CMD plane individually. For clarity, we show four representative CMDs, although all of the CMD planes listed in Table 6 were taken into account to obtain our final distance and reddening estimates. We now describe the individual empirical CMD comparisons before discussing the resultant mean distance and reddening values.

\subsection{Comparison Cluster Photometry}

We use differential-reddening corrected NGC 6544 photometry in all of our photometric comparisons to the various reference clusters so as to exploit the increase in photometric quality resulting from the application of our reddening maps (see Figure 5). As a consequence, all of the NGC 6544 photometry shown in Figure 11 is restricted to the ACS field of view.

The $\left(V, V-K_{S}\right)$ plane can be useful for obtaining a reddening estimate, since the large color baseline tends to increase the reddening sensitivity as compared to photometric errors. Additionally, Hendricks et al. (2012) point out that this plane has the somewhat unique advantage that the reddening vector is particularly insensitive to the assumed reddening law. To this end, in the upper left panel of Figure 11 we plot the result of matching the Rosenberg et al. (2000) $V$ and VVV $K_{S}$ photometry. To estimate the reddening, we look to M3 as the best candidate for a comparison, and match the Stetson CADC photometry to the near-infrared photometry of Valenti et al. (2004), which appears to be of somewhat higher quality than 2MASS. As an additional consistency check, we also include the RR Lyrae variables detected in M5 from Coppola et al. (2011). The reason we have performed this comparison using the ground-based $V$-band photometry rather than WFPC2 $F 555 \mathrm{~W}$ is due to the fact that it incorporates nearly the entire ACS WFC field of view and has a brighter saturation limit, and the lower photometric precision is thus compensated by the larger number of detectable stars on the RGB. We also show $\left(V, V-K_{S}\right)$ GGC fiducial sequences conveniently provided by Brasseur et al. (2010) for M13 and NGC 1851, the two clusters in that study that bracket the metallicity of NGC 6544. The VVV near-infrared photometry of NGC 6544 is compared with the M3 photometry from Valenti et al. (2004) in the upper right panel of Figure 11, although little information is gained in this case due to the reduced reddening sensitivity as well as the increased scatter in the M3 photometry compared to the color baseline.

Proceeding to the ACS photometry, we have overplotted CADC photometry of M72 (NGC 6981) as green points, converted from $B, R$ to $F 435 W, F 625 W$ colors using the coefficients given by Sirianni et al. (2005), as well as our M5 and M13 fiducial sequences from Section 4. Although these two clusters differ in metallicity from NGC 6544 by up to $\sim 0.15$ dex, the ACS WFC photometry is of similar quality in identical filters, and thus we can compare the portions of the CMD that are less metallicity sensitive (i.e., the unevolved main sequence and the RGB) as these sequences are insensitive to age anyway. We have also overplotted the individual star photometry for the M5 and M13 HBs, and using the M5 and M13 distance moduli and reddening from V13 (see Table 5), the blue HBs of the two clusters coincide nicely. Additionally, the $\mathrm{HBs}$ provide support for the distance and reddening we estimate for NGC 6544 based on the main sequence and RGB, as we achieve good simultaneous agreement between all of the principal evolutionary sequences.

Proceeding to the WFPC2 photometry, we overplot the M3 and M72 fiducial sequences from Section 4, again finding that the two sequences are essentially coincident, supporting the distance and reddening estimates of V13. However, as the photometry does not extend very far down the unevolved main sequence, we rely on the giant branch for our comparison with NGC 6544.

\subsection{Results}

A weighted mean of the values in Table 6 gives $(m-M)_{0}=$ $11.96 \pm 0.07\left(\sigma_{\bar{x}}\right)$ and $E(B-V)=0.79 \pm 0.01\left(\sigma_{\bar{x}}\right)$ under the assumption of a standard reddening law (we address this assumption below in Section 6). The scatter in the individual best-fit values is consistent with the individual observational uncertainties we have estimated, and is similar to that which persists in the reference cluster distance and reddening estimates (this is discussed further in Section 5.1). Furthermore, these mean values are in decent agreement with previous studies: analysis of the WFPC2 photometry (Piotto et al. 2002; Recio Blanco et al. 2005) via comparison to template clusters ${ }^{12}$ (including M3) found $E(B-V)=0.73$ and $(m-M)_{V}=$ 14.33, and Rosenberg et al. (2000) list $E(B-V)=0.74$ and $(m-M)_{V}=14.28$ from their ground-based photometry, yielding $(m-M)_{0}=12.07$ and 11.99 , respectively, assuming $R_{V}=3.1$. Valenti et al. (2010), using near-infrared photometric measurements of the RGB tip and slope, ${ }^{13}$ report

\footnotetext{
12 See http://www.astro.unipd.it/globulars.

13 See http://www.bo.astro.it/ GC/ir_archive.
} 

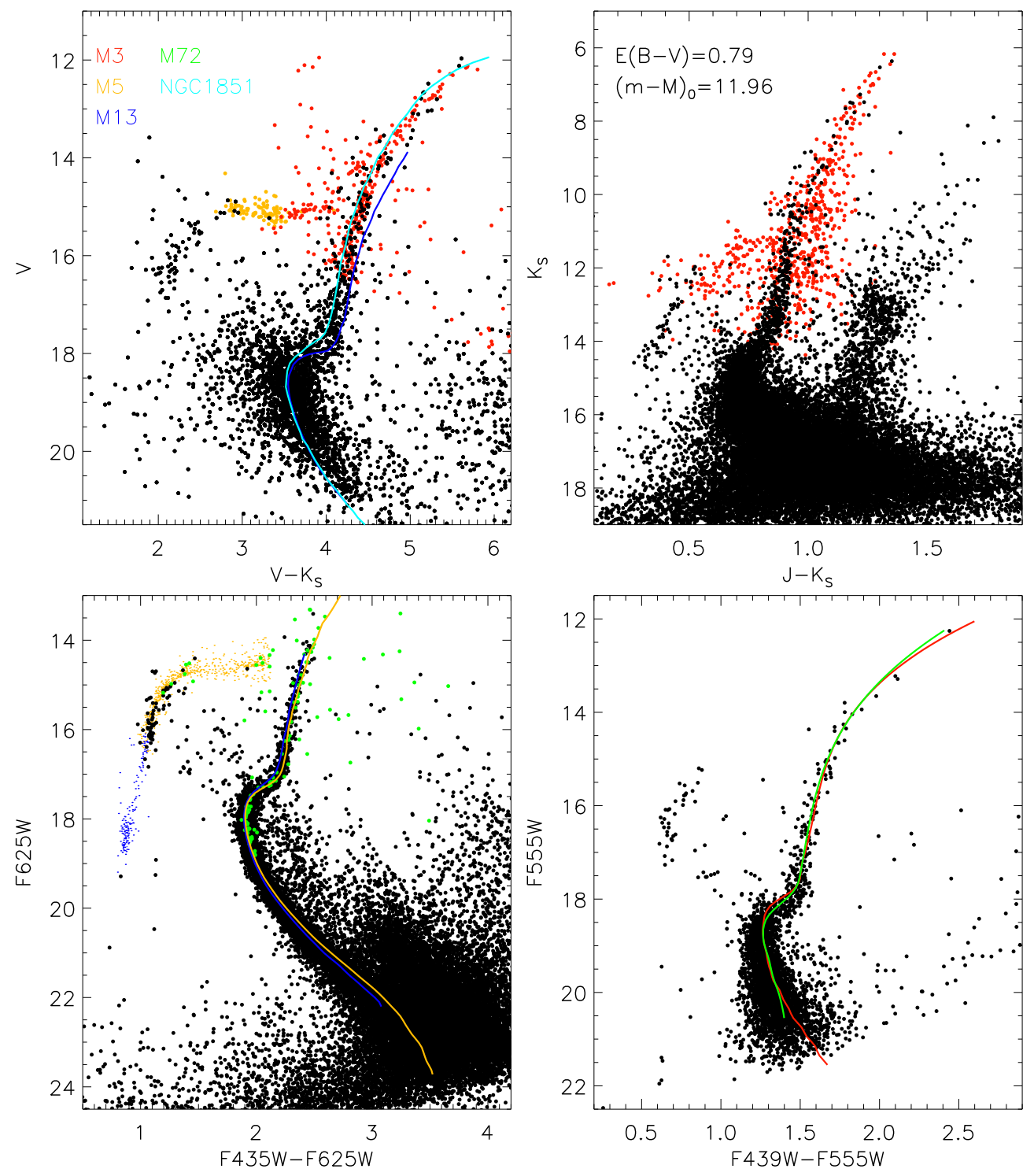

Figure 11. Photometry of NGC 6544 in four different color-magnitude planes (black dots), compared with photometry and fiducial sequences for various reference clusters at similar metallicity (see the text for details). The reference cluster photometry is color-coded by cluster as indicated in the upper left panel. In the upper right panel we give the estimated mean values of $E(B-V)$ and $(m-M)_{0}$ used in generating this figure. The assumed values of the reference cluster parameters are those of V13 given in Table 5.

$E(B-V)=0.78$ and $(m-M)_{0}=12.23$. None of these investigations could correct for differential reddening, and without exception, they all caution that this impacts the reliability of their results. Also, the zero point of our reddening map, determined by the location of the empirical fiducial sequences that we generate, is essentially arbitrary. While this zero point is likely to be representative of the value close to the center of the cluster since this is where the majority of the stars lie, the reddening map reveals that $E(B-V)$ varies by more than 0.2 just in the central arcminute of the cluster, and varies by a factor of $\Delta E(B-V) / E(B-V) \approx 0.5$ over the ACS WFC field of view. In light of this variation, the agreement both among previous studies and between their results and ours is encouraging.

\subsection{Some Caveats}

\subsubsection{The GGC Distance Scale}

We have chosen to determine the cluster distance and reddening via empirical comparisons to reference clusters, and so our results of course depend on the adopted reference cluster parameters. For consistency, we have adopted the values employed by V13 for all of the reference clusters, although recent studies imply that the GGC distance scale remains uncertain to more than 0.1 mag (e.g., Cohen \& Sarajedini 2012; V13). To examine this issue as it relates to our ensemble of comparison clusters, we compare in Figure 12 the distances, ages, and reddenings obtained by Dotter et al. (2010) and V13 as well as the [Fe/H] values employed by these two studies (extinction coefficients from Sirianni et al. 2005 were applied to the $(F 606 W, F 814 W)$ values in Dotter et al. 2010). This comparison is particularly instructive since these two studies employed the same photometry but obtained cluster parameters using different evolutionary models, color- $T_{\text {eff }}$ transformations, and methodologies. General trends with metallicity are evident, particularly in the case of the cluster distances, and Dotter et al. (2010) find ages that are systematically older, although the larger discrepancy at the metal-rich end could merely be a consequence of the different chemical abundances assumed by the two studies, shown in the bottom panel of Figure 12. While we do not intend to rehash the detailed comparison by V13 between their ages and those 


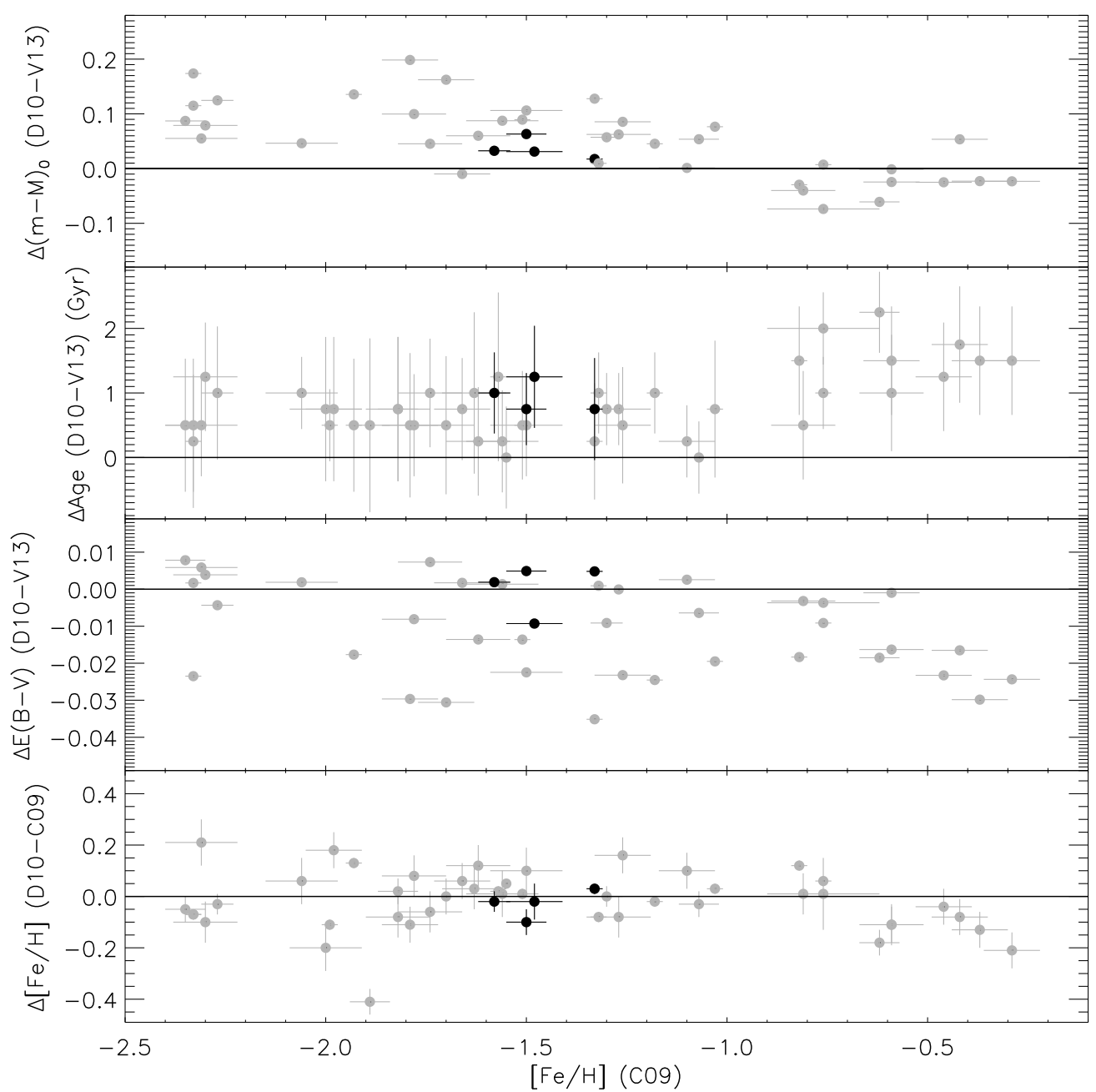

Figure 12. Comparison between $(m-M)_{0}$, age, $E(B-V)$, and $[\mathrm{Fe} / \mathrm{H}]$ values from Dotter et al. (2010) and $\mathrm{V} 13$ as a function of [Fe/H] from Carretta et al. (2009). All GGCs in common between the two studies are shown in gray, and those listed in Table 5 which we use as reference clusters are shown in black. The horizontal line in each plot indicates equality.

obtained from other studies, the implication of Figure 12 is that in spite of deep, precise, homogeneous photometry, the absolute systematic uncertainty that remains is on the order of $\sim 0.1 \mathrm{mag}$ in distance modulus and $\sim 1$ Gyr in age.

\subsubsection{Chemical Abundances}

Our empirical comparisons between NGC 6544 and the comparison clusters rest to some extent on the assumption that these clusters have similar abundances of not only $[\mathrm{Fe} / \mathrm{H}]$, but other metals and $\mathrm{He}$ as well. Importantly, clusterto-cluster abundance variations in these elements can affect the morphology of the observed evolutionary sequences used in Section 5.1 to estimate the distance and reddening. For example, DSED models (among others) imply that the location of the upper RGB is sensitive to $[\alpha / \mathrm{Fe}]$ as well as $[\mathrm{Fe} / \mathrm{H}]$ (Dotter et al. 2008). There is, in fact, observational evidence for abundance variations among some of our reference clusters. First, M13 may be significantly helium enhanced (Dalessandro et al. 2013; V13, but see Sandquist et al. 2010) while M3 may not (Dalessandro et al. 2013; Catelan et al. 2009). Furthermore, even though the blue HB of NGC 6544 is sparse, the difference in morphology as compared to the redder HBs of M3 and M72, which have identical values of $[\mathrm{Fe} / \mathrm{H}]$, is clear. Without commenting more generally on the vast and ongoing efforts to understand the parameters governing HB morphology (a review is given by Catelan 2009), recent spectroscopic studies imply that light element abundances play an important role in determining the photometric properties of GGC HBs (Marino et al. 2011, 2013). For these reasons, spectroscopy of stars along the red giant and HBs of NGC 6544 could confirm or deny whether the similarity in chemical abundance with the reference clusters extends beyond just $[\mathrm{Fe} / \mathrm{H}]$. We have excluded the subgiant branch from our photometric comparisons so as to keep our analysis independent of the various age determinations for these clusters, and this is doubly important since the morphology of the subgiant branch can be affected by abundances of CNO (D'Antona et al. 2009), potentially mimicking an age difference. However, Figure 20 of V13 suggests that at the metallicity of NGC 6544, relatively large changes in $\mathrm{CNO}(\Delta[\mathrm{O} / \mathrm{Fe}] \gtrsim 0.3 \mathrm{dex})$ or $\mathrm{He}$ $(\Delta Y \gtrsim 0.04)$ would be required to influence age measurements beyond their uncertainties. Lastly, the empirical photometric comparisons above have necessarily assumed a reddening law, and we now address this assumption.

\section{REDDENING LAW}

The assumption of a total to selective extinction ratio $R_{V}$, quantifying the dependence of extinction on wavelength, is needed to convert photometrically obtained cluster parameters such as distance modulus and extinction in a given bandpass to physical values. This issue is especially pertinent for clusters 


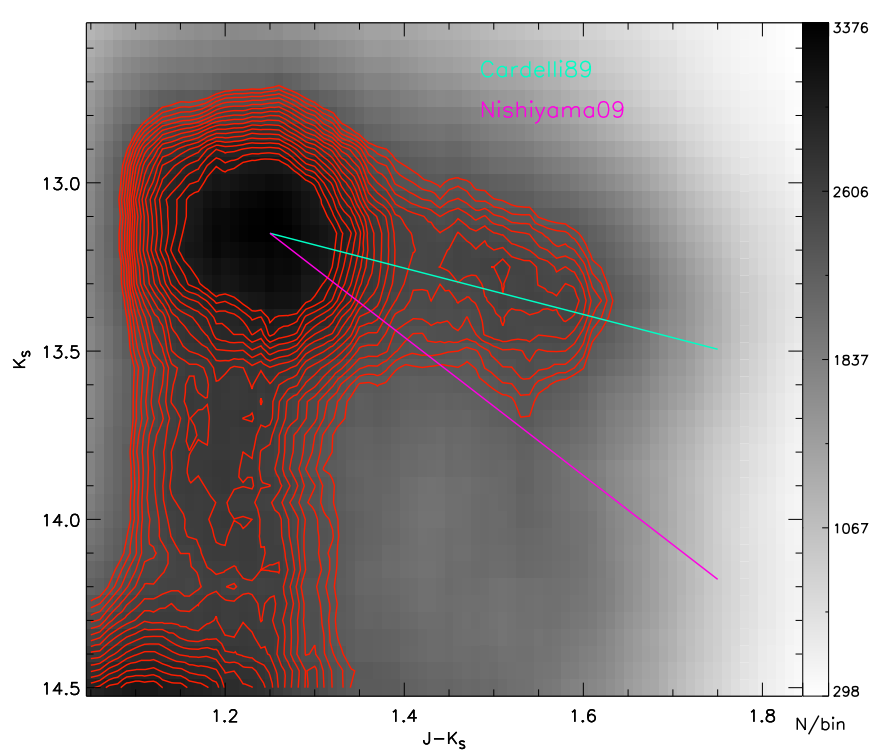

Figure 13. Color-magnitude density of Galactic bulge red clump stars in the direction of NGC 6544; see the text for details. The red lines are isodensity contours, and the cyan and magenta lines show the reddening vectors corresponding to a Cardelli et al. (1989) or Nishiyama et al. (2009) near-infrared reddening law, respectively.

located in the direction of the Galactic bulge, as there is strong evidence for variations in the reddening law at both optical (Nataf et al. 2013a) and infrared wavelengths (Nishiyama et al. 2009).

\subsection{Near-infrared Reddening Law}

The dependence of extinction on $R_{V}$ in the near-infrared was parameterized by Cardelli et al. (1989) using a powerlaw exponent of -1.61 , while recent studies by Nishiyama et al. (2009, and references therein) find that in the inner bulge $\left(|l| \lesssim 3^{\circ},|b| \lesssim 1^{\circ}\right)$, the reddening law in the near- and mid-infrared is well fit with a power-law exponent of -2.0 . NGC 6544 lies outside this region $(l=5.8365, b=-2.2024)$, but we may exploit the differential reddening observed over our large field of view to constrain the reddening law in the nearinfrared using the VVV photometry of the Galactic bulge red clump. In order to avoid the influence of cluster members, we examine only stars with $0.9<\left(J-K_{S}\right)<1.9,12<K_{S}<15$, and $R_{\text {clus }}>10^{\prime}$, although the exact location of this radial cut is not critical since the cluster and bulge RGBs are well separated in color in the near-infrared CMD (see Figure 2). We construct a Hess diagram within the aforementioned CMD limits by creating a grid of points spaced by 0.01 in $\left(J-K_{S}\right)$ color and 0.05 in $K_{S}$ magnitude, and for each grid point, we count the number of stars within 0.05 and $0.25 \mathrm{mag}$ in color and magnitude, respectively. The resulting Hess diagram is shown in Figure 13, and we have overplotted density contours in the interval between 0.7 and 0.9 times the difference between the maximum and minimum non-zero value, in steps of 0.015 times this value. A comparison between these observed contours and the Cardelli et al. (1989) and Nishiyama et al. (2009) reddening vectors reveals that at near-infrared wavelengths the observed differential reddening favors a Cardelli et al. (1989) law.

\subsection{Optical Reddening Law}

Nataf et al. (2013a) combined optical photometry from Optical Gravitational Lensing Experiment III (OGLE-III) with
Table 7

$A_{\Lambda} / A_{V}$ for HST ACS and WFPC2 Filters

\begin{tabular}{|c|c|c|c|c|c|}
\hline \multirow[t]{2}{*}{$R_{V}$} & \multicolumn{2}{|c|}{ ACS WFC } & \multicolumn{2}{|c|}{ WFPC2 } & \multirow[t]{2}{*}{ Reference } \\
\hline & $F 435 W$ & $F 625 W$ & $F 439 W$ & $F 555 W$ & \\
\hline 2.3 & 1.4527 & 0.8228 & 1.4686 & 1.0100 & This study \\
\hline 2.4 & 1.4341 & 0.8272 & 1.4492 & 1.0093 & \\
\hline 2.5 & 1.4170 & 0.8313 & 1.4313 & 1.0087 & \\
\hline 2.6 & 1.4011 & 0.8350 & 1.4147 & 1.0080 & \\
\hline 2.7 & 1.3865 & 0.8384 & 1.3994 & 1.0074 & \\
\hline 2.8 & 1.3728 & 0.8416 & 1.3851 & 1.0069 & \\
\hline 2.9 & 1.3601 & 0.8446 & 1.3718 & 1.0063 & \\
\hline 3.0 & 1.3482 & 0.8474 & 1.3594 & 1.0058 & \\
\hline 3.1 & 1.3370 & 0.8500 & 1.3478 & 1.0053 & \\
\hline 3.1 & & & 1.313 & 0.976 & Holtzman et al. $(1995)^{\mathrm{a}}$ \\
\hline 3.1 & 1.316 & 0.851 & & & Sirianni et al. $(2005)^{\mathrm{b}}$ \\
\hline 3.1 & 1.297 & 0.843 & & & Bedin et al. $(2005)^{\mathrm{c}}$ \\
\hline 3.1 & 1.354 & 0.862 & & & Bedin et al. $(2005)^{\mathrm{d}}$ \\
\hline
\end{tabular}

Notes.

a From Table 12(b) of Holtzman et al. (1995), which is based on a spectral type $\mathrm{K} 5$ spectrum and $R_{V}=3.1$.

b From Table 14 of Sirianni et al. (2005), for a spectral type G2 SED. The coefficients listed for spectral type M0 are $2.3 \%$ and $0.8 \%$ smaller for the $F 435 W$ and $F 625 W$ filters, respectively.

${ }^{\mathrm{c}}$ From Table 3 of Bedin et al. (2005), corresponding to a cool star $\left(T_{e}=4000 \mathrm{~K}\right.$, $\log g=4.5)$.

${ }^{\mathrm{d}}$ From Table 4 of Bedin et al. (2005), corresponding to a hot $\operatorname{star}\left(T_{e}=40,000 \mathrm{~K}\right.$, $\log g=4.5)$.

the near-infrared reddening maps of Gonzalez et al. (2012) to map the spatial variation of the reddening law over the OGLE-III field of view, parameterizing the results in terms of $R_{J K V I}=E\left(J-K_{S}\right) / E(V-I)$. The core of NGC 6544 unfortunately lies just outside the OGLE-III field of view, but the seven points in the Nataf et al. (2013a) maps within 20' of NGC 6544 (all to the southeast of the cluster center) have $R_{J K V I}$ values implying $2.3 \lesssim R_{V} \lesssim 2.7$. However, this line of evidence, as well as our conclusion above regarding the nearinfrared reddening law, has the limitation that it is based on measurements of bulge stars in the direction of NGC 6544, not of the cluster itself. Ideally, the value and uncertainty of $R_{V}$ can be well determined using cluster stars themselves (Hendricks et al. 2012; Campos et al. 2013), but these approaches require consistently deep precise photometry over a broad (optical-IR) wavelength range as well as reliable calibrating sequences and thus cannot presently be applied here. However, in light of the Nataf et al. (2013a) results, we wanted to explore the possibility that the optimal agreement between the NGC 6544 photometry and that of the comparison clusters could be reached by allowing a non-standard value of $R_{V}$. For this reason, we calculated values of the extinction coefficients $A_{\lambda} / E(B-V)$ as a function of $R_{V}$ for the four HST filters employed here, which are listed in Table 7, along with previous literature determinations for comparison.

The coefficients we calculate compare favorably with those from other studies, as the values corresponding to $R_{V}=3.1$ in the ACS WFC filters lie intermediate between those listed by Bedin et al. (2005) for a hot star and a cool star, in order to be applicable over the entire CMD as intended.

We can now attempt to constrain the optical reddening law using the empirical estimated color shifts in Table 6. We use an approach similar to that of Hendricks et al. (2012), where we choose a reference color (in this case we have chosen $V-K_{S}$ 

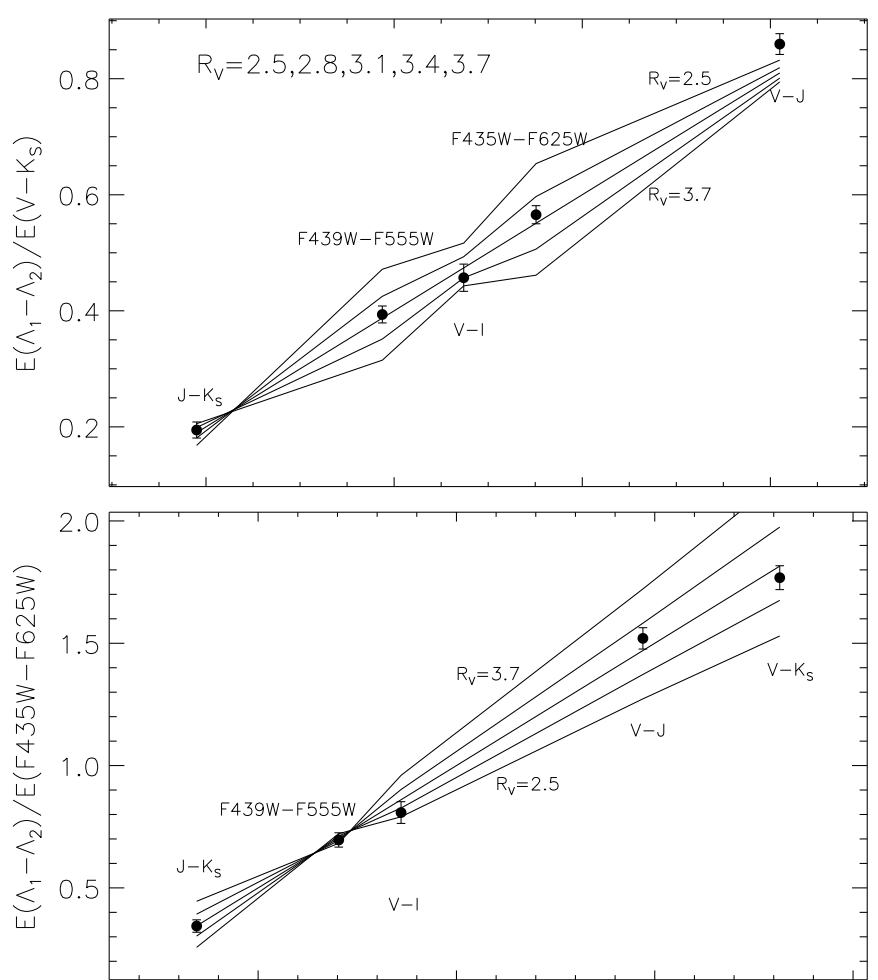

Figure 14. Empirically estimated color difference in several colors compared to a chosen reference color $\left(V-K_{S}\right)$ (top) or $(F 435 W-F 625 W)$ (bottom). The observed values and error bars are from Table 6, and each color is labeled and has been assigned an $x$-axis value so as to create a straight line when $R_{V}=3.1$. The lines show predicted values assuming $R_{V}=2.5,2.8,3.1,3.4,3.7$ and using the extinction coefficients from Table 7 for the $H S T$ filters and from Hendricks et al. (2012) for $V I J H K_{S}$ filters. The lines corresponding to $R_{V}=2.5$ and 3.7 are labeled in each plot.

due to its smaller relative uncertainty) and plot the ratio of the color shift in each filter pair $\Lambda_{1}-\Lambda_{2}$ to that of the reference color. In the top panel of Figure 14, we plot the equivalent of their $F_{\Lambda_{1}-\Lambda_{2}}$, equivalent to $E\left(\Lambda_{1}-\Lambda_{2}\right) / E\left(V-K_{S}\right)$ in our case, along with predictions based on different assumed values of $R_{V}$. Although the photometric quality of NGC 6544 as well as the comparison clusters limits the precision of any conclusions in some bandpasses, our estimates are generally consistent with a standard interstellar extinction law. The color shift we estimate in $V-J$ appears inconsistent with this conclusion, but not at a hugely significant level $(\sim 2 \sigma)$ in light of the quality of the available photometry, and as a check on our results we have repeated this procedure, but instead use the ACS $(F 435 W-F 625 W)$ color as a reference color (shown in the lower panel of Figure 14), validating at least to some extent our assumption of a standard reddening law for NGC 6544.

A complicating issue to be aware of is that filters close to the $B$ band, included in both our ACS and WFPC2 photometry, are particularly sensitive to the spectral energy distribution (SED) of the source (McCall 2004). More quantitatively, Hendricks et al. (2012, see their Tables 11-13 and Figures 11 and 12) show that over the range of stellar parameters sampled by our photometry, metallicity and total extinction only affect the extinction coefficients at a level of about $1 \%$. However, stellar surface gravity and temperature can each cause a change of $4 \%$ in $A_{B}$, and therefore could conspire to cause significant variations when simultaneously considering parameter space which includes the unevolved main sequence, the upper RGB, and the HB.

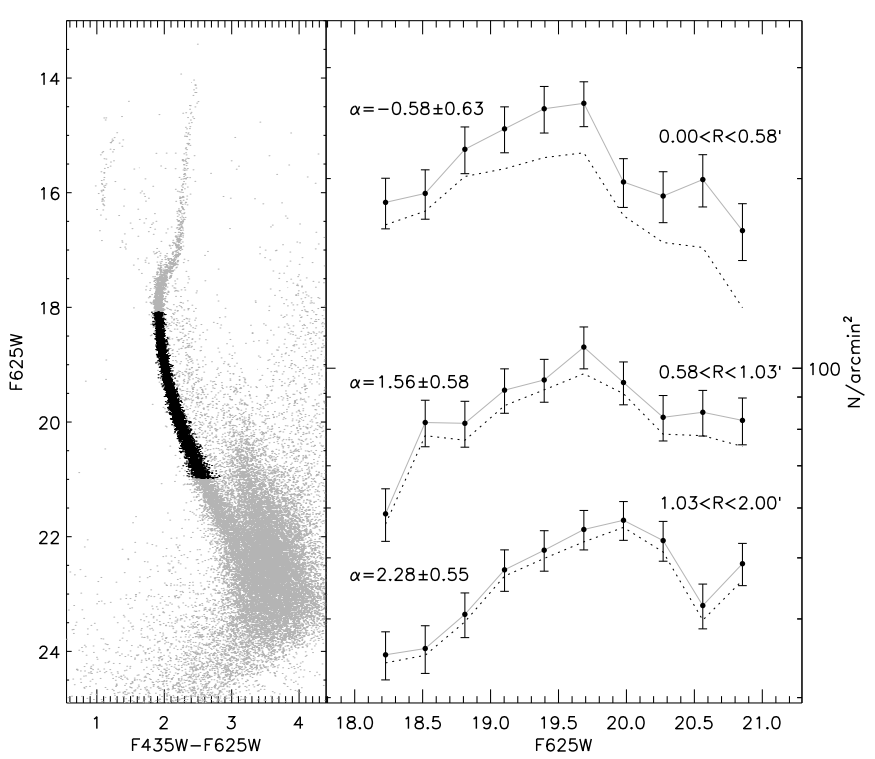

Figure 15. Left: differential reddening corrected ACS CMD, illustrating the stars selected to construct main sequence luminosity and mass functions. Right: luminosity functions in different radial zones, with the $y$-axis plotted on a logarithmic scale. Dashed lines show the LF obtained before completeness corrections, and the completeness-corrected LFs are shown as black points with error bars and connected with a gray line.

\section{LUMINOSITY AND MASS FUNCTIONS}

We construct cluster main sequence LFs from the differential reddening corrected ACS photometry to investigate the dynamical state of NGC 6544 via evidence of mass segregation. We build our LFs using stars from the observed main sequence turnoff faintward $(18.08<F 625 W<21)$ and within five times the standard deviation in color from the cluster fiducial sequence, shown in the left panel of Figure 15. In the right panel, we show LFs for three different radial zones, chosen so as to have an equal number of stars in each zone to achieve an optimal compromise between spatial resolution and Poissonian uncertainties on both the observed number of stars and the completeness corrections from the artificial star tests. Above each LF, we give the radial interval employed as well as the slope resulting from a power-law fit, illustrating that the relative fraction of faint stars decreases toward the cluster center. This result is unlikely to be due to contamination by field stars, and this can be quantified using our proper-motion selected sample from Section 3.3. Counting the number of proper-motion rejected stars in the CMD selection region used for the LF, applying completeness corrections, propagating their uncertainty and scaling by the relative areas, we find that the total predicted density of field stars contaminating the CMD selection region is $28 \pm 11 \mathrm{arcmin}^{-2}$. Since the difference between the brightest and faintest magnitude bins even in the outermost radial zone shown in Figure 15 is $\sim 20 \mathrm{arcmin}^{-2}$, the field star magnitude distribution would need to be severely biased toward bright magnitudes to significantly alter the observed trend in LF slope. Based on the observed color-magnitude distribution of field stars in Figure 6, there is no reason to expect that this would be the case.

Similarly flattened (as opposed to rising with decreasing mass) main sequence LFs have been observed in several other clusters with atypically flat power-law radial density profiles similar to that of NGC 6544 (see Section 8.2), and of these, Koch et al. (2004) found a similar trend with radius in their LFs 


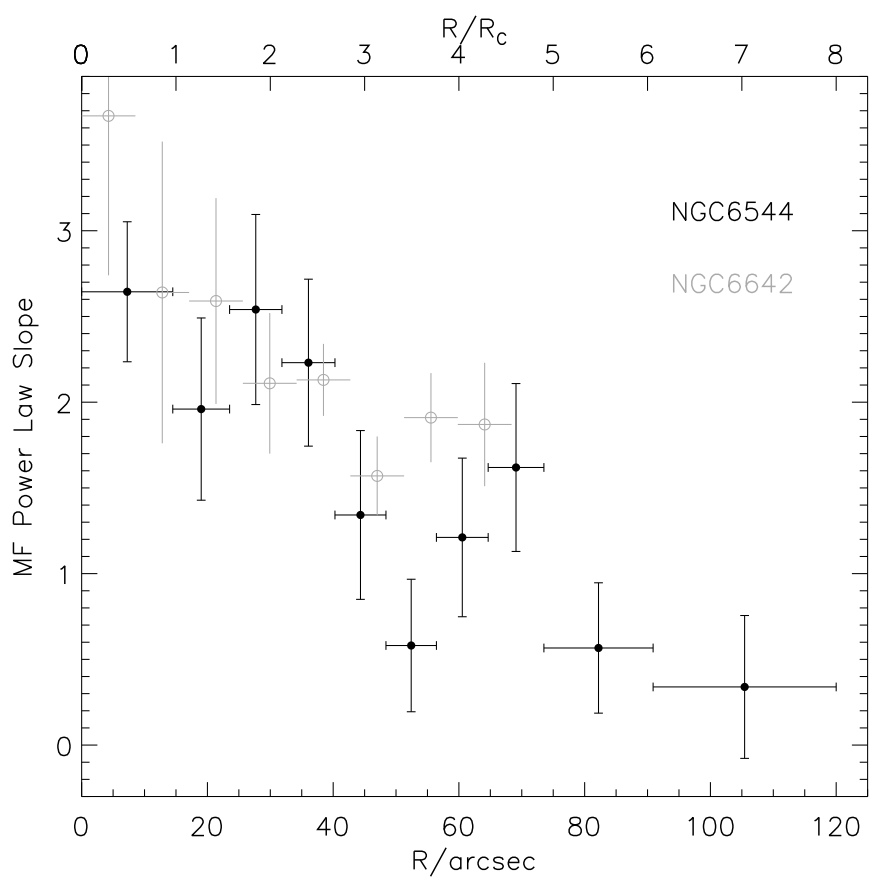

Figure 16. Mass function power-law slope obtained in 10 different radial bins, with the radial limits of each bin indicated by horizontal error bars. The vertical error bars indicate the standard deviation of the best-fit power-law exponents obtained over 1000 simulations in which the cluster distance modulus was randomly varied to propagate an uncertainty of $\sigma(m-M)=0.2 \mathrm{mag}$. The upper horizontal axis gives the radii in terms of the core radius from Section 8.2, and measurements for NGC 6642 from Balbinot et al. (2009) are overplotted in gray in terms of $R / R_{c}$ for comparison.

of Palomar 5. This has been interpreted as evidence of mass segregation, and tidal interaction in the case of Pal 5 since the less centrally concentrated lower mass stars would have been preferentially stripped.

To investigate this issue further, we convert the LFs to MFs, using the distance and reddening determined in Section 5.1, the reddening law from Section 6.2, and the DSED model corresponding to the observed cluster metallicity to convert observed magnitudes to stellar masses. We divide the main sequence stars selected for the LF analysis into mass bins that are evenly spaced in $\log M / M_{\odot}$, and fit a power law to the resulting MF in each of 10 radial zones, again chosen such that the number of stars in each zone is equal. We take the uncertainty in the cluster distance modulus into account using a bootstrapping procedure similar to that employed by Goldsbury et al. (2013), wherein the uncertainty of the MF slope in each radial bin is the standard deviation of the slope measured in that bin over 1000 iterations. In each of these iterations, a value of $(m-M)_{0}$ has been drawn from a Gaussian distribution centered on the value of 11.96 given in Section 5.1, and in light of the discussion in Section 5.4.1 we have conservatively used a standard deviation of $\sigma(m-M)=0.2$ for the distance modulus uncertainty. The completeness-corrected results, shown in Figure 16, illustrate that the observed present day MF of NGC 6544 is actually inverted close to the center of the cluster, and the relative deficiency of lower mass stars becomes more severe at smaller radial distances from the cluster center. A linear least-squares fit to the MF slope $\alpha$ as a function of radius from the cluster center in Figure 16 yields $(d \alpha / d r)=-0.0239 \pm 0.0048$ (where $r$ is in arcseconds), implying that despite the uncertainties, the inversion of the MF is statistically significant. Inverted MFs have been observed for a few other GCs, including NGC 6712
(Andreuzzi et al. 2001), NGC 2298 (di Marchi \& Pulone 2007), NGC 6366 (Paust et al. 2009), and AM-4 (Hamren et al. 2013). However, all of these clusters have an MF power-law slope that reaches a maximum value of $\alpha \lesssim 1$, so perhaps a better analog for NGC 6544 is NGC 6642 . The MF slope of this cluster reaches $\alpha>2$ inside its core radius, and its trend of LF turnover magnitude but also MF power-law slope with $R / R_{c}$ (Balbinot et al. 2009) is quite similar to what we observe for NGC 6544, as shown in Figure 16. To further investigate the structural parameters of NGC 6544, we now combine the $H S T$ and VVV photometry to construct a radial number density profile.

\section{STRUCTURAL PROPERTIES}

\subsection{Redetermining the Cluster Center}

It was apparent from the ACS and WFPC2 imaging that the cluster center given in the Harris (1996, 2010 edition) catalog, taken from Shawl \& White (1986), as well as the value given in the SIMBAD database (different by 0.'82 in right ascension (R.A.) from the Harris catalog value) was somewhat erroneous. We used the ACS photometry to redetermine the cluster center by fitting ellipses to density contours. The use of stellar number counts rather than integrated light has the advantage that it is insensitive to stochastic variations caused by the most luminous stars (Anderson et al. 2010), and the centers yielded by the concentric ellipse fits are a more sensitive quantifier of the true cluster center than using pairs of azimuthally opposite "pie slices" to test for asymmetries (Goldsbury et al. 2010). The procedure we follow is virtually identical to that employed in Anderson et al. (2010) and Goldsbury et al. (2010), and consists of the following steps: first, a density map is constructed, using spatial grid points spaced by $2^{\prime \prime}$ and centered on the Harris catalog value of the cluster center. In accord with the aforementioned two studies, we find that both a magnitude cut and spatial overbinning are necessary to create a sufficiently smooth density map. Thus, we use only stars with $F 625 W<21$ to construct the map, and for each grid point, the density value is the number of stars within a box of width $20^{\prime \prime}$ centered on the grid point. All grid points within a box width of the edge of a chip are excluded so as not to influence the density contours. Next, eight density contours are generated, spaced evenly between the minimum non-zero value and the maximum value of the density. Finally, a nonlinear least-squares fitting of an ellipse to the four innermost density contours is performed, again excluding any points on each contour which fall in the gap between the WFC chips. The fitted ellipse center $(\Delta$ R.A. $\cos \delta, \Delta$ decl.) from each of these four contours is taken as an individual measurement of the cluster center, and the scatter in these values is used to characterize the precision of the measured centers. We found that reliable values could also be obtained making limiting magnitude cuts at $F 625 W=19,20$, and 22 as well for a total of 16 measurements of the cluster center. An example of the isodensity contour fits from the ACS photometry with $F 625 \leqslant 21$ is shown in the left panel of Figure 17 along with the old and new cluster center locations. The resulting cluster center we have used throughout our analysis, obtained by averaging all of the individual fit ellipse centers, is R.A. $=271.833832=18: 07: 20.119$, decl. $=$ $-24.998223=-24: 59: 53.60(\sigma=0.95)$, which is compared with the previous values in Table 8 .

Unfortunately, this procedure cannot be performed on either the WFPC2 or VVV photometry due to their somewhat lower photometric quality as well as inhomogeneous spatial coverage 

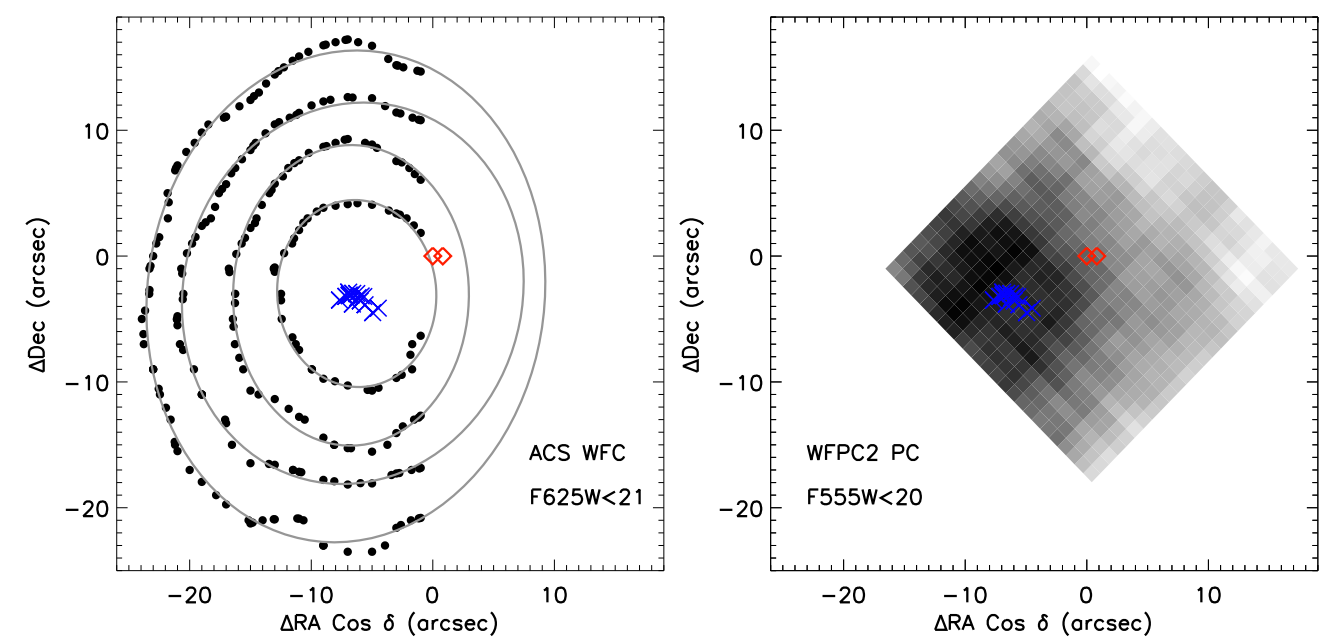

Figure 17. Left: an example of the four innermost isodensity contours (black points) constructed from a density map of the ACS WFC field of view, in this case using stars with $F 625 \mathrm{~W}<21$. These are used to determine the cluster center by fitting ellipses (gray lines), and each individual center measurement resulting from an ellipse fit is shown as a cross. For comparison, previous literature values of the cluster center are shown as diamonds (see the text). Right: our individual center measurements and the literature centers overplotted on a density map of the WFPC2 PC chip, shown at the same spatial scale of the left-hand panel for comparison.

(A color version of this figure is available in the online journal.)

Table 8

Coordinates of NGC 6544 Center

\begin{tabular}{lccc}
\hline \hline $\begin{array}{l}\text { R.A. (J2000) } \\
\text { (hh:mm:ss.ss) }\end{array}$ & $\begin{array}{c}\text { Decl. (J2000) } \\
\text { (dd:mm:ss.s) }\end{array}$ & $\begin{array}{c}\text { Uncertainty } \\
\text { (arcsec) }\end{array}$ & Reference \\
\hline 18:07:20.58 & $-24: 59: 50.4$ & 0.5 & Shawl \& White (1986) \\
18:07:20.64 & $-24: 59: 50.4$ & & SIMBAD \\
$\mathbf{1 8 : 0 7 : 2 0 . 1 2}$ & $\mathbf{- 2 4 : 5 9 : 5 3 . 6}$ & $\mathbf{0 . 9 5}$ & This study \\
\hline
\end{tabular}

in the case of the former and central incompleteness from overcrowding in the case of the latter. However, for visual comparison, we show the old and new values of the cluster center on a density map of the WFPC2 PC chip in the righthand panel of Figure 17 as well.

\subsection{Projected Number Density Profile}

\subsubsection{Observed Profile}

With an accurate location of the cluster center in hand, we may now construct a cluster (projected) radial density profile, simultaneously exploiting the spatial resolution of $H S T$, and the wide field available from the VVV survey. We construct this profile using stellar number densities, which we denote as $\Sigma(r)$, rather than integrated light since Goldsbury et al. (2013) have demonstrated the pitfalls of obtaining cluster structural parameters from integrated light profiles due to their assumption of a constant mass-to-light ratio. Since that study also illustrated the dependence of cluster structural parameters on stellar mass, we need to ensure that the same mass range is being sampled when matching profiles from multiple sources of photometry. Therefore we set the limits on the luminosity range used to obtain the density profile as follows: the faint end of the sampled luminosity range is determined from the VVV photometry, which becomes crowding limited close to the cluster center. We want to maximize the radial overlap between the VVV and ACS density profiles to match them, while ensuring that incompleteness is not affecting the portion of the VVV profile that is matched to the ACS profile. While we are in the process of modifying our PSF photometry pipeline to quantify the photometric incompleteness of the VVV photometry via extremely time consuming artificial star tests, in the meantime the examination of VVV $K_{S}$ LFs at different radii from the cluster center implies that with a faint limit at $K_{S}=16$, the VVV photometry should be complete outside a clustercentric radius of $r=1$ '.4. We use the ACS $90 \%$ completeness limit to set the upper (bright) cutoff of the sampled luminosity range since completeness drops off quite sharply due to saturation, as shown in Figure 3. Thus, to compute the observed number density profile, we used stars in the magnitude ranges $15<F 625 \mathrm{~W}<$ 19.5 and $11.5<K_{S}<16$ for the ACS and VVV photometry, respectively. To convert the faint magnitude limit in the $K_{S}$ filter to the ACS $F 625 \mathrm{~W}$ filter (and vice versa for the bright limit) while ensuring the an identical mass range was being sampled in both sets of photometry, we determined the masses corresponding to the bright (in $F 625 \mathrm{~W}$ ) and faint (in $K_{S}$ ) magnitude limits using a DSED isochrone with $[\mathrm{Fe} / \mathrm{H}]=-1.5$, $[\alpha / \mathrm{Fe}]=0.4$, and the best-fit distance and reddening from Section 5.1, finding a stellar mass range of $0.7<M<0.8 M_{\odot}$ for the stars used to construct our number density profiles. To sample the full radial extent of the cluster, the VVV- and ACS-based number density profiles were combined by matching in the radial range $1^{\prime} .4<r<2^{\prime}$ from the cluster center. Specifically, the VVV profile was interpolated in radius to also include the (radial) locations in the ACS profile and vice versa so that both profiles contained number density values (and uncertainties) for all data points present in either profile within the aforementioned range of radii from the cluster center. A (Poissonian) weighted mean scale factor between the two profiles was then calculated as an average across all of these radial locations. In order to achieve the best compromise between Poissonian uncertainties and spatial resolution of the profile, especially at small radii, radial bins were spaced in proportion to $r^{x}$, with $3<x<5$ depending on the source of the photometry. The total uncertainty in each radial bin is the quadrature sum of the Poissonian uncertainties from the observed number of stars as well as from artificial star tests in the case of the HST photometry.

\subsubsection{Profile Fitting}

We describe briefly the various types of analytical density profiles which we fit to our observations. An excellent discussion of GGC radial profiles can be found in McLaughlin \& van der 


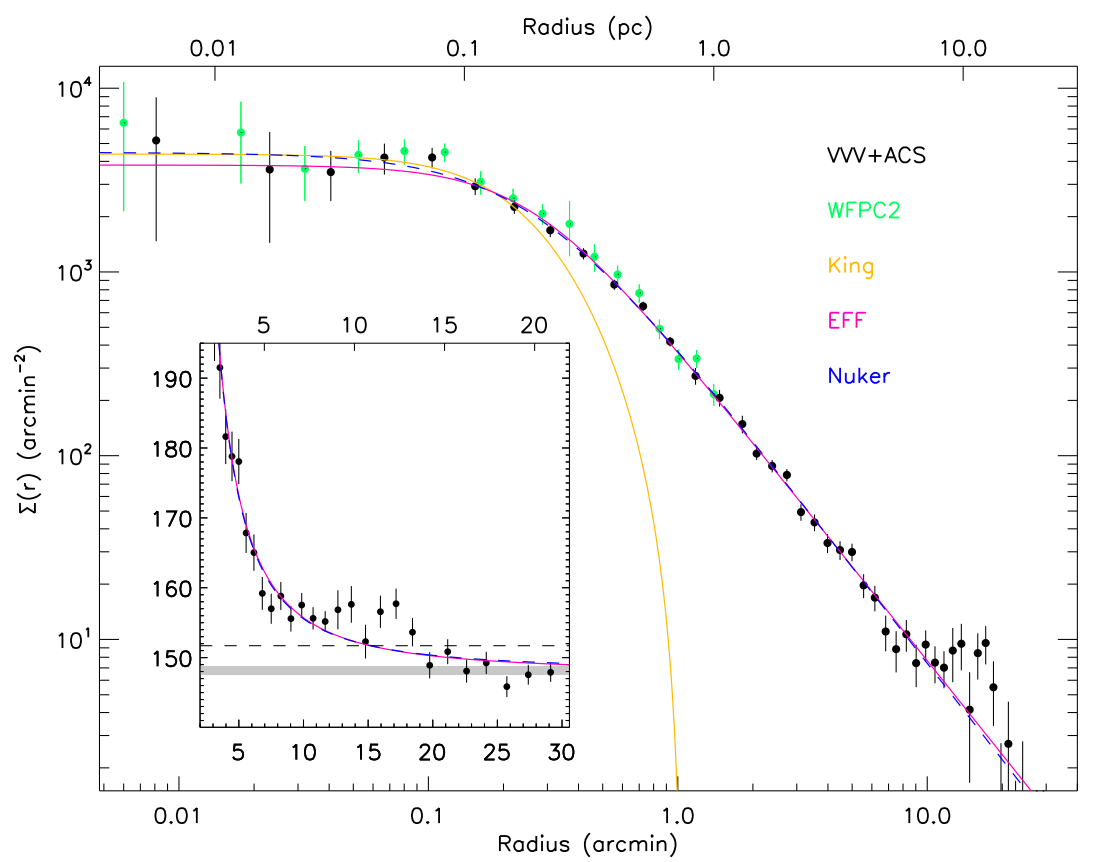

Figure 18. Background-subtracted radial number density profile and analytical fits. The observed matched VVV+ACS profile is shown with filled black circles and error bars, and the number density profile from the WFPC2 photometry is shown for comparison in green. Analytical King, EFF, and Nuker fits are shown in orange, magenta, and blue, respectively. The inset highlights the outer portion of the profile before background subtraction on a linear rather than logarithmic scale, and there the observed mean background density and its standard deviation is shown as a gray shaded region, and the dotted line indicates the value predicted by the Besancon Galaxy model. For both plots, the upper horizontal axis gives radii in parsec assuming $(m-M)_{0}=11.96$ from Section 5.1.

(Supplemental data of this figure are available in the online journal.)

Table 9

Radial Density Profile Parameters

\begin{tabular}{|c|c|c|c|c|c|c|c|}
\hline \multirow[t]{2}{*}{ Template } & \multirow{2}{*}{$\begin{array}{c}b^{\mathrm{a}} \\
\left(\operatorname{arcmin}^{-2}\right)\end{array}$} & \multicolumn{2}{|c|}{$R_{b}$} & \multicolumn{2}{|c|}{$R_{\mathrm{hl}}$} & \multirow[t]{2}{*}{$\chi^{2}$} & \multirow[t]{2}{*}{$\eta$} \\
\hline & & $(\operatorname{arcmin})$ & $(\mathrm{pc})^{\mathrm{b}}$ & $(\operatorname{arcmin})$ & $(\mathrm{pc})^{\mathrm{b}}$ & & \\
\hline $\mathrm{EFF}$ & $147.8 \pm 0.6$ & $0.25 \pm 0.02$ & $0.18 \pm 0.02$ & $0.33 \pm 0.01$ & $0.23 \pm 0.02$ & 1.26 & $1.69 \pm 0.03$ \\
\hline Nuker & $148.1 \pm 0.7$ & $0.27 \pm 0.07$ & $0.21 \pm 0.03$ & $0.36 \pm 0.03$ & $0.29 \pm 0.03$ & 1.31 & $1.71 \pm 0.06$ \\
\hline $\mathrm{King}^{\mathrm{c}}$ & & 0.29 & 0.21 & 0.14 & 0.10 & & \\
\hline
\end{tabular}

Notes.

${ }^{\text {a }}$ Background constant resulting from fits to non-background-subtracted profile.

b Assuming $(m-M)_{0}=11.96$ from Section 5.1.

${ }^{\mathrm{c}}$ Parameters obtained fitting the background-subtracted profile at $r<0$ '.25; see the text.

Marel (2005), and a summary and description of the functional forms of various analytical template profiles is given by Küpper et al. (2010). We initially perform fits that include the constant background density $b$, as this allows a comparison between the value of $b$ resulting from the fit and our observed background density of $\Sigma_{b}=148.1 \pm 0.6$ which we measure at $R>19^{\prime}$. This value compares reasonably well to the $\Sigma_{b}=151.7$ predicted by the Besancon Galaxy model (Robin et al. 2003) to the extent typical of GGCs in the direction of the bulge (e.g., Alonso Garcia et al. 2012). Next, we subtract our observed background value, propagating its uncertainty, and perform the fits again on the background-subtracted profile, shown in Figure 18. For comparison, we also show the profile from the WFPC2 photometry as green points, having selected stars in the same mass range as used for the ACS and VVV profiles by requiring $15.8<F 555 W<20$. The resulting best-fit parameters and reduced $\chi^{2}$ values are given in Table 9. In all cases, we performed our fits to the matched VVV+ACS number density profile using a Levenberg-Marquardt nonlinear least-squares procedure, fitting the following analytical template forms.
1. An analytical King (1962) profile:

$$
\Sigma(r)=k\left[\frac{1}{\sqrt{1+\left(R / R_{b}\right)^{2}}}-\frac{1}{\sqrt{1+\left(R_{t} / R_{b}\right)^{2}}}\right]^{2}+b .
$$

As discussed extensively by McLaughlin \& van der Marel (2005), the imposition of a strict outer cutoff radius can cause this profile to fit observations poorly, and so we include other analytical templates that lack this restriction.

2. The EFF profile (Elson, Fall, and Freeman; Elson et al. 1987) is comprised of a flat core, transitioning at a break radius $R_{b}$ to a power-law dropoff in the outer regions of the cluster characterized by the exponent $\eta$, and no strict cluster edge radius: ${ }^{14}$

$$
\Sigma(r)=k\left[1+\left(\frac{R}{R_{b}}\right)^{2}\right]^{-\eta / 2}+b .
$$

\footnotetext{
14 The outer power-law slope notated here and in Küpper et al. (2010) as $\eta$ is commonly notated as $\gamma$, and Carballo-Bello et al. (2012) point out that in the case of McLaughlin \& van der Marel (2005), our $\eta$ corresponds to their $\gamma-1$.
} 
3. The Nuker template of Lauer et al. (1995) is similar to the EFF profile in that it also falls off as a power law at large radii, but has the added flexibility that it does not require a flat core:

$$
\Sigma(r)=k 2^{\frac{\eta-\gamma}{\alpha}}\left[\frac{R}{R_{b}}\right]^{-\gamma}\left[1+\left(\frac{R}{R_{b}}\right)^{\alpha}\right]^{-\frac{\eta-\gamma}{\alpha}}+b .
$$

Here, $\gamma$ represents the power-law slope of the core, and $\alpha$ dictates the smoothness of the transition between the two slopes $\eta$ and $\gamma$ near $R_{b}$.

We also attempted to fit the more flexible templates of Küpper et al. (2010) and van der Marel \& Anderson (2010) but were unable to obtain reliable fits, likely due to their larger number of free parameters. Given the apparent power-law nature of the outer region of this cluster, we could not obtain reliable parameters for the King profile fit, with or without the background term. However, for comparison, we illustrate the King profile resulting from a fit to the background-subtracted profile inside the EFF break radius of 0 '.25, which yields $R_{b}=$ 0 '.29 (the King-defined "core radius") and $R_{t}=1$ '.05. Regarding nomenclature, we have used the notation $R_{b}$ to refer to the characteristic break radius (in the case of the Nuker and EFF profiles) or core radius as defined in Equation (2) for the King profile, reserving a consistent definition of $R_{c}$ as the radius at which the number density reaches half of its central value.

\subsubsection{Results}

The EFF profile resulted in the fit with the lowest reduced $\chi^{2}$ value, and numerical integration of this profile yields a half light radius of $R_{\mathrm{hl}}=0.33$. To convert the angular sizes, which we measure from the best-fit analytical profiles, to physical sizes while propagating an uncertainty in the cluster distance, we perform a bootstrapping procedure similar to that employed in Section 7. For each of 1000 iterations, the assumed cluster distance modulus is drawn from a Gaussian distribution with a mean $(m-M)_{0}=11.96$ and standard deviation of $\sigma(m-M)_{0}=$ 0.2 in order to calculate the physical distances corresponding to the resulting best-fit profile parameters. The uncertainties of the cluster parameters in parsec reported in Table 9 are the standard deviations calculated over these 1000 iterations.

The resulting physical sizes corresponding to $R_{c}$ and $R_{\mathrm{hl}}$ are 0.18 and $0.23 \mathrm{pc}$, respectively, although these are projected values. Chatterjee et al. (2013) is the latest study to point out the discrepancy between observed two-dimensional projected quantities and the physical three-dimensional quantities to which they correspond, finding that their three-dimensional (code defined, in their case) core radius and half mass radius are typically a factor of $\sim 1.5-2$ larger than their observational analogs, albeit with considerable variation (see their Figures 5 and 6). Conveniently, for these quantities, they also compare the distributions they obtain from a grid of simulations to observed values for GGCs. According to their Figure 6, our observed $R_{\mathrm{hl}}$ would imply a half mass radius of $\sim 0.4 \mathrm{pc}$ for NGC 6544, still quite small. Curiously, Figures 8 and 9 of Chatterjee et al. (2013), which include a comparison to observed GGC properties, suggest somewhat contradictory results based on our observed core and half light radii: the small core radius predicts a relatively high central density compared to GGCs as an ensemble, whereas the ratio of core to half light radius predicts the opposite. The latter also implies that NGC 6544 is probably not core collapsed, and while the core power-law slope $\gamma=0.004 \pm 0.316$ from the Nuker fit is consistent with a flat core (required by the EFF profile), uncertainties do not allow us to completely rule out the possibility that this cluster is core collapsed and has a core profile that is not flat (e.g., Noyola \& Gebhardt 2006, but also see Miocchi et al. 2013). Also, the presence of two known pulsars in NGC 6544, one of which likely has a massive white dwarf or neutron star companion (Lynch et al. 2012, and references therein) could be seen as evidence for a high central density.

\subsubsection{Evidence of Tidal Interaction}

While our results do not necessarily permit firm constraints on the nature of the cluster core, perhaps the most striking feature of Figure 18 is the power-law slope extending out to $\sim 40 R_{c}$. There is now a fairly extensive subset of GGCs that have outer surface brightness profiles well represented by a power law, in many cases better than a King profile (McLaughlin \& van der Marel 2005; Carballo-Bello et al. 2012). However, our value of $\eta=1.7$ for the outer power-law slope is quite small compared to the range observed in these two broad, systematic studies, which found $\eta>2$ for all GGCs they analyzed. Meanwhile, power-law slopes of $\eta \sim 1.8$ have been observed for a handful of GGCs: AM 4 (Carraro 2009), Whiting 1 (Carraro et al. 2007), Pal 5 (Koch et al. 2004), and Pal 13 (Côté et al. 2002). In comparison to NGC 6544, these clusters are all much more distant, likely less luminous, and more strikingly, have low central concentrations of $c<1$. For comparison, $R_{t}=19^{\prime}$ and $R_{c}=0.25$ gives $c=$ 1.88 for NGC 6544, and we return again to the analogy with NGC 6642, which is similarly concentrated. The analogy seems to extend even further, as both clusters are also moderately metal-poor, have sparse blue HBs, and a centrally concentrated blue straggler population. As NGC 6642 lies in much closer physical proximity to the bulge, one possibility is that these are two physically similar clusters being viewed at different points in their orbits, which could explain why they are similar in virtually all respects except for the outer portions of their radial density profiles.

On the theoretical side, models have shown that a power-law slope, and various sub-features, such as breaks in this slope, can result from tidal stripping due to gravitational interaction with the Milky Way potential (e.g., Johnston et al. 1999, 2002). More recently, Küpper et al. (2010) simulated the effects of such interaction on GGCs to investigate the resulting morphologies of their tidal tails. Along these lines, perhaps the most plausible explanation for the fact that NGC 6544 is fairly concentrated yet exhibits quite a flat outer power-law slope is given by Küpper et al. (2011). Through a grid of $N$-body simulations, they find that the appearance of a cluster radial density profile (and its observed velocity dispersion) can depend on the orbital phase of the cluster. More specifically, the deceleration of a cluster when approaching apogalacticon causes material lost via tidal stripping to enhance the observed density in the cluster outskirts. In this context, the flat power-law slope we observe could have two implications: first, that NGC 6544 had undergone tidal stripping due to interactions with the Milky Way potential, perhaps similarly to the aforementioned four clusters with $\eta \approx 1.8$; and second, that it may be near apogalacticon on a noncircular orbit. Importantly, Küpper et al. (2010) find that even on circular orbits, an observed power-law slope can be a natural consequence of tidal stripping. However, the flatness of the slope we observe for NGC 6544 suggests that it is a relatively extreme case, and perhaps further simulations could address whether this slope could be generated without invoking orbital eccentricity. Also, the apparent overdensity of stars observed at $12^{\prime}<r<19^{\prime}$ 
(shown on a linear scale in the inset of Figure 18) bears some similarity to features visible in the observed GGC profiles of Carballo-Bello et al. (2012) as well as the bulge cluster VVV CL002 (Moni Bidin et al. 2011), which similarly could not be fit by a King profile. Interestingly, similar features are also seen in the simulation results shown in Figure 15 of Johnston et al. (1999) and Figure 6 of Johnston et al. (2002). This provides further support for the idea that NGC 6544 has undergone tidal stripping via interaction with the Milky Way, consistent with the flattened luminosity and MFs described in Section 7.

\subsubsection{Comparison To Literature Values}

The structural parameters we measure differ vastly from those published by Trager et al. (1995), who list $\left(R_{c}, R_{h}, R_{t}\right)=$ $(0.05,1 ' 21,2$ '.05). However, in that study the authors make quite clear on multiple occasions that the parameters that they derive for NGC 6544 are almost certainly untrustworthy. First, they state that errors in the determination of the cluster center from integrated light can cause artifacts in the resulting surface brightness profiles. They go on to mention that the observed brightness profiles of NGC 6544 and Pal 12 were fit with a higher order $(n=6)$ Chebyshev polynomial than all other clusters in their study, cautioning that the bump seen in their profile of Pal 12 (which is also seen only in NGC 6544 and NGC 7492) has "little physicality." And finally, for NGC 6544, they note that the parameters resulting from a King profile fit are dubious based on the fact that the fit half light radius is a large fraction of the fit tidal radius. Given this due diligence on the part of Trager et al., it should be unsurprising that the cluster structural parameters differ significantly from their reported values, and this is one reason why we have not attempted to scale their (integrated light) profile to match ours. However, roughly a decade before the Trager et al. (1995) study, Webbink (1985) listed $R_{c}=$ $0.35, R_{t}=8.8$, and $d=2.6 \mathrm{kpc}$, remarkably consistent with what we observe given their use of different assumptions for the reddening law, the distance to the Galactic center, and a King (1966) surface brightness profile to derive their structural parameters. ${ }^{15}$

\section{SUMMARY AND FUTURE DIRECTIONS}

We summarize our principal results as follows.

1. A high spatial resolution differential reddening map generated over the inner region of the cluster reveals that, as with other GGCs located in the direction of the Galactic bulge, reddening variations on a spatial scale of arcseconds are present, and in this case are quite severe $(\Delta E(B-V) / E(B-V) \approx 0.5)$. This map is fairly insensitive to the assumed reddening law at a level of $\sim 0.01 \mathrm{mag}$ in optical bandpasses. The zero point of our reddening map corresponds to a Gonzalez et al. (2012) value (based on the bulge red clump) of $E\left(J-K_{S}\right)=0.814 \pm 0.046$.

2. We have estimated the distance, reddening, and age of NGC 6544 by empirical comparison to clusters with similar metallicities, estimating $(m-M)_{0}=11.96, E(B-V)=0.79$ using the semi-empirical zero-age horizontal branch-based scale of V13. We also find that NGC 6544 is likely coeval with M13, although given the relevant uncertainties we cannot exclude the possibility that it is as young as M5. In the context of the GGC age-metallicity relation, this leaves some ambiguity as

\footnotetext{
15 In fact, the observations Webbink (1985) used to obtain these parameters are from Kron et al. (1984), which Trager et al. (1995) downweighted to zero in favor of more recent CCD photometry.
}

to whether NGC 6544 belongs to the disk or halo population pending further kinematic studies.

3. We have redetermined the center of NGC 6544 to a precision of $\sim 1^{\prime \prime}$, finding a positional offset of $\sim 7^{\prime \prime}$ from literature values. Using the newly determined cluster center, we fit a radial number density profile to NGC 6544 over its entire radial extent, finding that the cluster may have a radial extent as large as $\sim 19^{\prime}$. The observed radial number density profile cannot be fit with an analytical King model, and is best fit by a power law with an unusually flat slope of 1.7. This value, in combination with density enhancements seen in the outer profile, a flattened main sequence LF and inverted MF, suggest that NGC 6544 is mass segregated and has lost stars via tidal interaction with the gravitational potential of the Milky Way.

Several interesting questions have now been raised regarding NGC 6544 which could be addressed on observational and/or theoretical grounds. First, additional deep high spatial resolution photometry could allow a more detailed study of the profile of the cluster core and possibly more quantitative constraints on the reddening law. Also, given the proximity of NGC 6544, relative proper motions over a wider field of view could then be used to isolate the cluster population down to lower masses, facilitating a detailed study of mass segregation, especially if radial velocities were to be obtained spectroscopically. Ideally, absolute proper motions could eventually be used to obtain an orbit, allowing a direct test of the hypothesis that the flatness of the power-law surface brightness profile is related to orbital eccentricity, although this may be quite challenging observationally given the high density of bulge stars in the background. Meanwhile, the most viable avenue for corroboration of the cluster distance and reddening may be time series photometry of classical pulsators such as RR Lyrae and SX Phe stars.

The VVV survey may be able to fulfill many of these goals in the future as it accumulates time series imaging in the $K_{S}$ band. For example, its wide-field nature could allow the isolation of cluster members via relative proper motions over the entire radial extent of the cluster. Meanwhile, in the context of pulsating variables, investigations of period-luminosity relations in the near-infrared have the advantage that they are much less susceptible to effects of evolutionary and chemical abundance variations than at optical wavelengths (e.g., Catelan et al. 2004; Alonso-García et al. 2013).

We wish to thank A. Dolphin for his kind technical support in our use of DOLPHOT, as well as the anonymous referee for thoughtful comments and suggestions. R.E.C. gratefully acknowledges financial support from Fondo GEMINI-CONICYT 32100008 and F.M. is thankful for financial support from FONDECYT for project 3140177. D.G., F.M., and R.E.C. also acknowledge financial support from the Chilean BASAL Centro de Excelencia en Astrofisica y Tecnologias Afines (CATA) grant PFB-06/2007. Based on observations made with the NASA/ESA Hubble Space Telescope, obtained from the data archive at the Space Telescope Science Institute. STScI is operated by the Association of Universities for Research in Astronomy, Inc. under NASA contract NAS 5-26555. This research has made use of the facilities of the Canadian Astronomy Data Centre operated by the National Research Council of Canada with the support of the Canadian Space Agency, as well as the SIMBAD database, operated at CDS, Strasbourg, France. This publication makes use of data products from the Two Micron All Sky Survey, which is a joint project of the 
University of Massachusetts and the Infrared Processing and Analysis Center/California Institute of Technology, funded by the National Aeronautics and Space Administration and the National Science Foundation.

\section{REFERENCES}

Alcaino, G. 1983, A\&AS, 52, 105

Alonso-García, J., Dékány, I., Catelan, M., Contreras Ramos, R., \& Minniti, D. 2013, arXiv:1307.0419

Alonso Garcia, J., Mateo, M., Sen, B., et al. 2012, AJ, 143, 70

Anderson, J., \& King, I. R. 2003, PASP, 115, 113

Anderson, J., Sarajedini, A., Bedin, L. R., et al. 2008, AJ, 135, 2055

Anderson, J., \& van der Marel, R. P. 2010, ApJ, 710, 1032

Andreuzzi, G., de Marchi, G., Ferraro, F. R., et al. 2001, A\&A, 372, 851

Balbinot, E., Santiago, B. X., Bica, E., \& Bonatto, C. 2009, MNRAS, 396, 1596

Bedin, L. R., Cassisi, S., Castell, F., et al. 2005, MNRAS, 357, 1038

Bonatto, C., \& Bica, E. 2007, MNRAS, 377, 1301

Bonatto, C., \& Bica, E. 2010, A\&A, 516, A81

Bonatto, C., Bica, E., \& Girardi, L. 2004, A\&A, 415, 571

Bono, G., Stetson, P. B., VandenBerg, D. A., et al. 2010, ApJL, 708, L74

Brasseur, C. M., Stetson, P. B., VandenBerg, D. A., et al. 2010, AJ, 140, 1672

Campos, F., Kepler, S. O., Bonatto, C., \& Ducati, J. R. 2013, MNRAS, 433, 243

Carballo-Bello, J. A., Gieles, M., Sollima, A., et al. 2012, MNRAS, 419, 14

Cardelli, J. A., Clayton, G. C., \& Mathis, J. S. 1989, ApJ, 345, 245

Carraro, G. 2009, AJ, 137, 3809

Carraro, G., Zinn, R., \& Moni Bidin, C. 2007, A\&A, 466, 181

Carretta, E., Bragaglia, A., Gratton, R., D’Orazi, V., \& Lucatello, S. 2009, A\&A, 508,695

Catelan, M. 2009, Ap\&SS, 320, 261

Catelan, M., Grundahl, F., Sweigart, A. V., Valcarce, A. A. R., \& Cortés, C. 2009, ApJL, 695, L97

Catelan, M., Minniti, D., Lucas, P. W., et al. 2011, in RR Lyrae Stars, MetalPoor Stars, and the Galaxy, ed. A. McWilliam (Carnegie Observatories Astrophysics Series, Vol. 5; Pasadena, CA: The Observatories of the Carnegie Institution of Washington), 145

Catelan, M., Pritzl, B. J., \& Smith, H. A. 2004, ApJS, 154, 633

Chatterjee, S., Umbreit, S., Fregeau, J. M., \& Rasio, F. A. 2013, MNRAS, 429, 2881

Chené, A.-N., Borissova, J., Clarke, J. R. A., et al. 2012, A\&A, 545, A54

Chun, S.-H., Kim, J.-W., Shin, I.-G., et al. 2010, A\&A, 518, 15

Cohen, R. E., \& Sarajedini, A. 2012, MNRAS, 419, 342

Coppola, G., Dall'Ora, M., Ripepi, V., et al. 2011, MNRAS, 416, 1056

Côté, P., Djorgovski, S. G., Meylan, G., Castro, S., \& McCarthy, J. K. 2002, ApJ, 574,783

Dalessandro, E., Salaris, M., Ferraro, F. R., Mucciarelli, A., \& Cassisi, S. 2013, MNRAS, 430, 459

D’Antona, F., Stetson, P. B., Ventura, P., et al. 2009, MNRAS, 399, L151

di Marchi, G., \& Pulone, L. 2007, A\&A, 467, 107

Dolphin, A. E. 2000, PASP, 112, 1383

Dotter, A., Chaboyer, B., Jevremovic, D., et al. 2008, ApJS, 178, 89

Dotter, A., Sarajedini, A., Ander, J., et al. 2010, ApJ, 708, 698

Elson, R. A. W., Fall, M. S., \& Freeman, K. C. 1987, ApJ, 323, 54

Emerson, J., McPherson, A., \& Sutherland, W. 2006, Msngr, 126, 41

Ferraro, F. R., Valenti, E., \& Origlia, L. 2006, ApJ, 649, 243

Goldsbury, R., Heyl, J., \& Richer, H. 2013, ApJ, 778, 57

Goldsbury, R., Richer, H. B., Anderson, J., et al. 2010, AJ, 140, 1830

Gonzaga, S., et al. 2011, ACS Data Handbook (Version 6.0; Baltimore: STScI)

Gonzalez, O. A., Rejkuba, M., Zoccali, M., et al. 2012, A\&A, 543, 13

Hamren, K. M., Smith, G. H., Guhathakurta, P., et al. 2013, AJ, 146, 116

Harris, W. E. 1996, AJ, 112, 1487

Hazen, M. L. 1993, AJ, 106, 1055
Hendricks, B., Stetson, P. B., VandenBerg, D. A., \& Dall'Ora, M. 2012, AJ, 144,25

Holtzman, J. A., Burrows, C. J., Casertano, S., et al. 1995, PASP, 107, 1065

Johnston, K. V., Choi, P. I., \& Guhathakurta, P. 2002, AJ, 124, 127

Johnston, K. V., Sigurdsson, S., \& Hernquist, L. 1999, MNRAS, 302, 771

King, I. R. 1962, AJ, 67, 471

King, I. R. 1966, AJ, 71, 64

Koch, A., Grebel, E. K., Odenkirchen, M., Martínez-Delgado, D., \& Caldwell, J. A. R. 2004, AJ, 128, 2274

Kron, G. E., Hewitt, A. V., \& Wasserman, L. H. 1984, PASP, 96, 198

Küpper, A. H. W., Kroupa, P., Baumgardt, H., \& Heggie, D. C. 2010, MNRAS, 407, 2241

Küpper, A. H. W., Mieske, S., \& Kroupa, P. 2011, MNRAS, 413, 863

Landolt, A. U. 1992, AJ, 104, 340

Lauer, T. R., Ajhar, E. A., Byun, Y.-I., et al. 1995, AJ, 110, 2622

Lynch, R. S., Freire, P. C. C., Ransom, S. M., \& Jacoby, B. A. 2012, ApJ, 745, 109

Marin-Franch, A., Aparicio, A., Piotto, G., et al. 2009, ApJ, 694, 1498

Marino, A. F., Milone, A. P., \& Lind, K. 2013, ApJ, 768, 27

Marino, A. F., Villanova, S., Miline, A. P., et al. 2011, ApJL, 730, L16

Massari, D., Mucciarelli, A., Dalessandro, E., et al. 2012, ApJL, 755, L32

Mauro, F., Moni Bidin, C., Chené, A.-N., et al. 2013, RMxAA, 49, 189

McCall, M. L. 2004, AJ, 128, 2144

McLaughlin, D. E., \& van der Marel, R. P. 2005, ApJS, 161, 304

Milone, A. P., Piotto, G., Bedin, L. R., et al. 2012, A\&A, 540, 16

Minniti, D., Lucas, P. W., Emerson, J. P., et al. 2010, NewA, 15, 433

Miocchi, P., Lanzoni, B., Ferraroet, F. R., et al. 2013, ApJ, 774, 151

Moni Bidin, C., Mauro, F., Geisler, D., et al. 2011, A\&A, 535, A33

Nataf, D. M., Gould, A., Fouqué, P., et al. 2013a, ApJ, 769, 88

Nataf, D. M., Gould, A. P., Pinsonneault, M. H., \& Udalski, A. 2013b, ApJ, 766,77

Nishiyama, S., Tamura, M., Hatano, H., et al. 2009, ApJ, 696, 1407

Noyola, E., \& Gebhardt, K. 2006, AJ, 132, 447

Paust, N. E. Q., Aparicio, A., Piotto, G., et al. 2009, AJ, 137, 246

Piotto, G., King, I. R., Djorgovski, S. G., et al. 2002, A\&A, 391, 945

Recio-Blanco, A., Piotto, G., de Angeli, F., et al. 2005, A\&A, 432, 851

Robin, A. C., Reylé, C., Derrière, S., \& Picaud, S. 2003, A\&A, 409, 523

Rosenberg, A., Piotto, G., Saviane, I., \& Aparicio, A. 2000, A\&AS, 144, 5

Saito, R. K., Hempel, M., Minniti, D., et al. 2012, A\&A, 537, 107

Salaris, M., Chieffi, A., \& Straniero, O. 1993, ApJ, 414, 580

Sandquist, E. L., Gordon, M., Levine, D., \& Bolte, M. 2010, AJ, 139, 2374

Sarajedini, A. 2009, in IAU Symp. 258, The Ages of Stars, ed. E. E. Mamajek, D. R. Soderblom, \& R. F. G. Wyse (Cambridge: Cambridge Univ. Press), 221

Sarajedini, A., Bedin, L. R., Chaboyer, B., et al. 2007, AJ, 133, 1658

Sarajedini, A., \& Demarque, P. 1990, ApJ, 365, 219

Saviane, I., da Costa, G. S., Held, E. V., et al. 2012, A\&A, 540, 27

Shawl, S. J., \& White, R. E. 1986, AJ, 91, 312

Sirianni, M., Jee, M. J., Benítez, N., et al. 2005, PASP, 117, 1049

Skrutskie, M. F., Cutri, R. M., Stiening, R., et al. 2006, AJ, 131, 1163

Stetson, P. B. 1987, PASP, 99, 191

Stetson, P. B. 1994, PASP, 106, 250

Trager, S. C., King, I. R., \& Djorgivski, S. 1995, AJ, 109, 218

Valenti, E., Ferraro, F. R., \& Origlia, L. 2010, MNRAS, 402, 1729

Valenti, E., Ferraro, F. R., Perina, S., \& Origlia, L. 2004, A\&A, 419, 139

VandenBerg, D. A., Bolte, M., \& Stetson, P. B. 1990, AJ, 100, 445

VandenBerg, D. A., Brogaard, K., Leaman, R., \& Casagrande, L. 2013, ApJ, 775,134

van der Marel, R. P., \& Anderson, J. 2010, ApJ, 710, 1063

von Braun, K., \& Mateo, M. 2001, AJ, 121, 1522

Webbink, R. F. 1985, in Dynamics of Star Clusters, ed. J. Goodman \& P. Hut (Dordrecht: Reidel), 541 